\title{
50 Jahre Keltenmuseum Hallein. Fundort - Forschung - Vermittlung
}

\author{
Holger Wendling
}

\begin{abstract}
Zusammenfassung
Im Jahr 2020 feierte das Keltenmuseum Hallein sein 50-jähriges Jubiläum. Als eines der bedeutendsten archäologischen Fundortmuseen Europas zeigt es die spektakulären Funde der Salzmetropole auf dem Dürrnberg, die im Netzwerk der europäischen Eisenzeit als wirtschaftliche Drehscheibe und kultureller Impulsgeber diente. Die bis zu 2500 Jahre alten Relikte aus Siedlungen, Gräbern und Salzbergwerken stehen seit jeher im Fokus der „Archäologie der Kelten“ und sind programmatischer Namensgeber des 1970 eröffneten Museums. Seine Wurzeln gehen indes bis in die Tage des ersten bildungsbürgerlichen Engagements für Lokalgeschichte und Archäologie zurück. Als Halleiner Heimatmuseum und erstes Museum im Land Salzburg in den 1830er-Jahren etabliert, war die Institution bis in das 20. Jh. vor allem der jüngeren Geschichte der Salinenstadt verpflichtet und entwickelte sich erst seit den 1950er-Jahren zum archäologischen $\mathrm{Mu}$ seums- und Forschungszentrum, das heute inoffiziell als Salzburger Zentralmuseum für Urgeschichte firmiert. Als Forschungsinstitution in der Tradition des Österreichischen Forschungszentrums Dürrnberg verfolgt die mit dem Museum assoziierte Dürrnbergforschung internationale und interdisziplinäre wissenschaftliche Aufarbeitungsprojekte und Forschungsinitiativen in der Gräber- und Siedlungsarchäologie sowie der Archäometrie.
\end{abstract}

\section{Schlüsselbegriffe}

Museum, Institutionsgeschichte, Kelten, Eisenzeit, Salzbergbau, Forschung, Archäologie.

\footnotetext{
Abstract - 50 Years of Keltenmuseum Hallein. Site - Research Education

In 2020, the Keltenmuseum Hallein celebrated its 50th anniversary. As one of the most important archaeological site museums in Europe, it exhibits the spectacular finds of the Dürnberg salt metropolis, which served as an economic centre and cultural catalyst in the network of Iron Age Europe. The relics from settlements, graves and salt mines, which are up to 2,700 years old, have always been the focus of an 'Archaeology of the Celts' and are thus the programmatic eponym of the museum, which opened in 1970 . Its roots, however, go back to the days when the educated middle-classes first developed an interest in local history and archaeology. Established in the 1830s as Hallein's museum of local history, until the $20^{\text {th }}$ century the institution was mainly dedicated to the medieval and more recent history of the salt-mining town. It was only in the 1950s that it developed into
}

an archaeological museum and research centre, today unofficially known as Salzburg's central museum of prehistory. As a research institution in the tradition of the Austrian Research Centre Dürrnberg, the Dürnberg Research Department, which is associated with and located at the museum, pursues international and interdisciplinary scientific projects and initiatives in burial and settlement archaeology, and archaeometry.

\section{Keywords}

Museum, institutional history, Celts, Iron Age, salt mining, research, archaeology.

\section{Heimatmuseum und archäologische Liebhaberei}

Als sich im Oktober 2020 die Gründung des Keltenmuseum Hallein zum 50. Mal jährte, war dies uneingeschränkter Grund zur Freude und erhoffter Anlass eines „Jubiläumsjahres“. Dass dieses Festjahr in das 51. Jahr hineinreicht, ja erst heuer begangen werden kann, ist den Auswirkungen der Covid-19-Pandemie zuzuschreiben, soll aber der Freude keinen Abbruch tun. Das aufgeschobene Jubiläum, das 2020 nur in „kleinstem Rahmen“ gefeiert werden konnte, sowie die von vielerlei Seite schmerzlich vermissten Möglichkeiten physischen Erlebens und sozialer Interaktion im Museum lassen die identitätsstiftende und soziale Rolle von Kultur, Museum und Wissenschaft in der Postmoderne des 21. Jhs. umso deutlicher hervortreten. Vor diesem Hintergrund ist das 50-jährige Jubiläum des Keltenmuseum Hallein nicht nur ein runder Geburtstag sondern auch die Feier eines Lebensabschnitts, dem in gewiss anderer Qualität annähernd 150 Jahre Halleiner Museumsgeschichte vorangingen. Gelegenheit, zurückzublicken, die Gegenwart zu würdigen, aber auch eine Perspektive für die Zukunft zu bieten.

Es ist ein merkwürdiger Zufall, dass die Gründung des ältesten Museums im Land Salzburg, des Heimatmuseums Hallein, und die erste schriftliche Studie zur Archäologie des Dürrnbergs in dasselbe Jahr fallen. Implizit markierte jenes Jahr 1832 schon vor beinahe 200 Jahren, dass Hallein 
und sein Salzwesen mit den Relikten einer schon damals als „keltisch“ angesprochenen vorrömischen Besiedlung auf das Innigste verbunden ist. ${ }^{1}$ Schon weit älter sind die ersten Nachrichten über archäologische Funde auf und im Berg, über die Franz Dückher von Haslau in seiner „Saltzburgischen Chronica“ von 1666 berichtet. $^{2}$ Die beiden im 16. und 17. Jh. zusammen mit altem Gezähe gefundenen „Männer im Salz“ sind der Forschung verloren, nur Dückhers Beschreibung zeichnet ein sehr anschauliches Bild vom Zustand der Salzmumie aus dem Jahr 1573: „Im Salzberg Türrnberg 6300 Schuh tieff im ganzen Berg ein Mann 9. Spannen lang mit Fleisch, Bein, Haar, Bart und Kleidung ganz unverwesen, jedoch etwas breit zusammen geschlagen, am Fleisch ganz geselcht, gelb und hart wie ein Stockfisch, ausgehaut worden. ${ }^{3}$ Kaum abzusehen, welche Erkenntnisse aus den Mumien jener Opfer eisenzeitlicher Grubenunglücke mit modernen Analysemethoden zu ziehen gewesen wären, wäre man nicht gezwungen gewesen, sie aufgrund ästhetisch-olfaktorischer Umstände „nachzubestatten“.

So fanden weder diese frühen noch spätere Relikte des reichen archäologischen Erbes auf und am Fuße des Halleiner Hausberges Eingang in die Bestände der noch jungen, 1832 initiierten Sammlung, die über Jahrzehnte ihrer Gründungsphase an Finanznot, räumlicher Einschränkung sowie dem Fehlen eines verantwortlichen Kustos litt. ${ }^{4}$ Die 1813 beim Abbruch des Mauttores geretteten Requisiten der Bürgergarde wurden zunächst in der hiernach benannten „Zeugkammer“ im dritten Stock des Rathauses verwahrt. Obwohl schon seit 1832 als Städtisches Museum benannt, wurden die Objekte erst 1843 der Öffentlichkeit zugänglich gemacht. ${ }^{5}$ In den folgenden Dekaden musste die Sammlung mehrfach umziehen und gelangte vom Rathaus in das Bürgerspital, das Feuerwehrdepot im neuen Stadtteil Burgfried, retour in das Bürgerspital und schließlich wiederum in die Zeugkammer. ${ }^{6}$ Die in unregelmäßiger Folge bestellten ehrenamtlichen Kustoden legten das Hauptaugenmerk der Sammlungstätigkeit auf die reichen mittelalterlich-neuzeitlichen Quellen, die in Form von Realien der Volkskultur, des Zunft- und Innungswesens, von Gemälden und Skulpturen

1 Zur archäologischen Forschungsgeschichte des Dürrnbergs: Moosleitner 1977. - Penninger 1980. - Zeller 2001, 71-80. Wendling 2013. - Wendling 2016. - Zur Geschichte des Keltenmuseum Hallein: Penninger 1963. - Penninger 1972, 28-30. - PenNINGER 1976.

2 Willvonseder 1931. - Klein 1961. - Schatteiner, Stöllner 2001.

3 DǗchher von Haslau 1666, 263.

4 Penninger 1972, 28.

5 Penninger 1963, 4. - Penninger 1976, 41.

6 WAGNER 1913, 4. und nicht zuletzt durch Archivalien die Stadt- und Salinengeschichte an der Tennengauer Salzach illustrierten. ${ }^{7}$ Unter diesen Gegenständen fanden sich einige, die schon weit früher, zu Zeiten des ersten „Mannes im Salz“ erfasst wurden - freilich nicht in musealer Absicht. Am 23. Februar 1570 führte man minutiös Buch über den Bestand des Amtshauses des Salinenpflegers (Abb. 1). Die offizielle Registratur anlässlich der Übergabe der Amtsgeschäfte an den nachfolgenden Beamten, die Unterschlagung verhinderte und der Bestandssicherung diente, nennt Leitern, Feuerhaken und Gewichte, „1 Alltes Speiß Kässtl“, das Henkersseil (?) und diverse Hand- und Fußschellen „sambt den Khettn darinn man die Gefanng fuerdt“. Da einige der Gegenstände später im Bestand des Stadtmuseums auftauchen, steht das Inventar des Salinenamtes in der Halleiner „Pfleg“, dem heutigen Sitz des Keltenmuseums in gewisser und zweifacher Weise am Beginn der musealen Inventarisierung in Hallein.

Jahrhunderte später und weit nach Gründung des Heimatmuseums war es der Initiative des Heimatforschers und Lokalpatrioten Sebastian Wimmer (1843-1905) und des Bürgermeisters Alois Oedl (1842-1898) zu verdanken, dass 1882 die Zeugkammer als wirkliches „Städtisches Museum“ etabliert wurde. ${ }^{8}$ Ein zwischenzeitlich vom Direktor des Salzburger Museums, Alexander Petter (1832-1905) gefasster Plan, ebendort in der benachbarten Provinzhauptstadt „den Halleiner Musealschätzen im Museum Carolino-Augusteum [...] einen gesonderten Platz einzuräumen “ wurde nicht umgesetzt. ${ }^{9}$

Die während des 19. Jhs. vereinzelt auf dem Dürrnberg - das Kurbad war bis 1938 freilich noch eine eigenständige Gemeinde - geborgenen archäologischen Funde verloren sich vereinzelt im Antikenhandel und in Privatsammlungen oder gelangten glücklicherweise in die archäologische Sammlung des damaligen Vaterländischen bzw. Städtischen Museums Carolino-Augusteum in Salzburg. ${ }^{10}$ Hier finden sich bis heute die Fundstücke und Grabungsdokumentation, die der gebürtige Halleiner Johann A. Seethaler (17621844) 1831/32 in seinen Manuskripten der „allerersten Celtischen und Römischen Alterthümer am Dürrnberg und zu Hallein an der Salza in Verbindung mit ihren Salinen " dem bildungsbürgerlichen Publikum bekannt machte. ${ }^{11}$ Auch im Archivbestand des Keltenmuseums findet sich ein Exemplar jener Schrift, das eine der frühesten archäologischen „Befundzeichnungen“ birgt, die von heimatforschenden

\footnotetext{
7 Penninger 1972, 28-29. - Zuletzt Koller 2016.

8 Kanzler 1912, 154-155. - Penninger 1976, 41. - Wagner 1913, 2.

9 Wagner 1906a, 5. - WAGNER 1913, 4.

10 WeNDLING 2013, 61.

11 Seethaler 1831. - Sonntag 2000.
} 

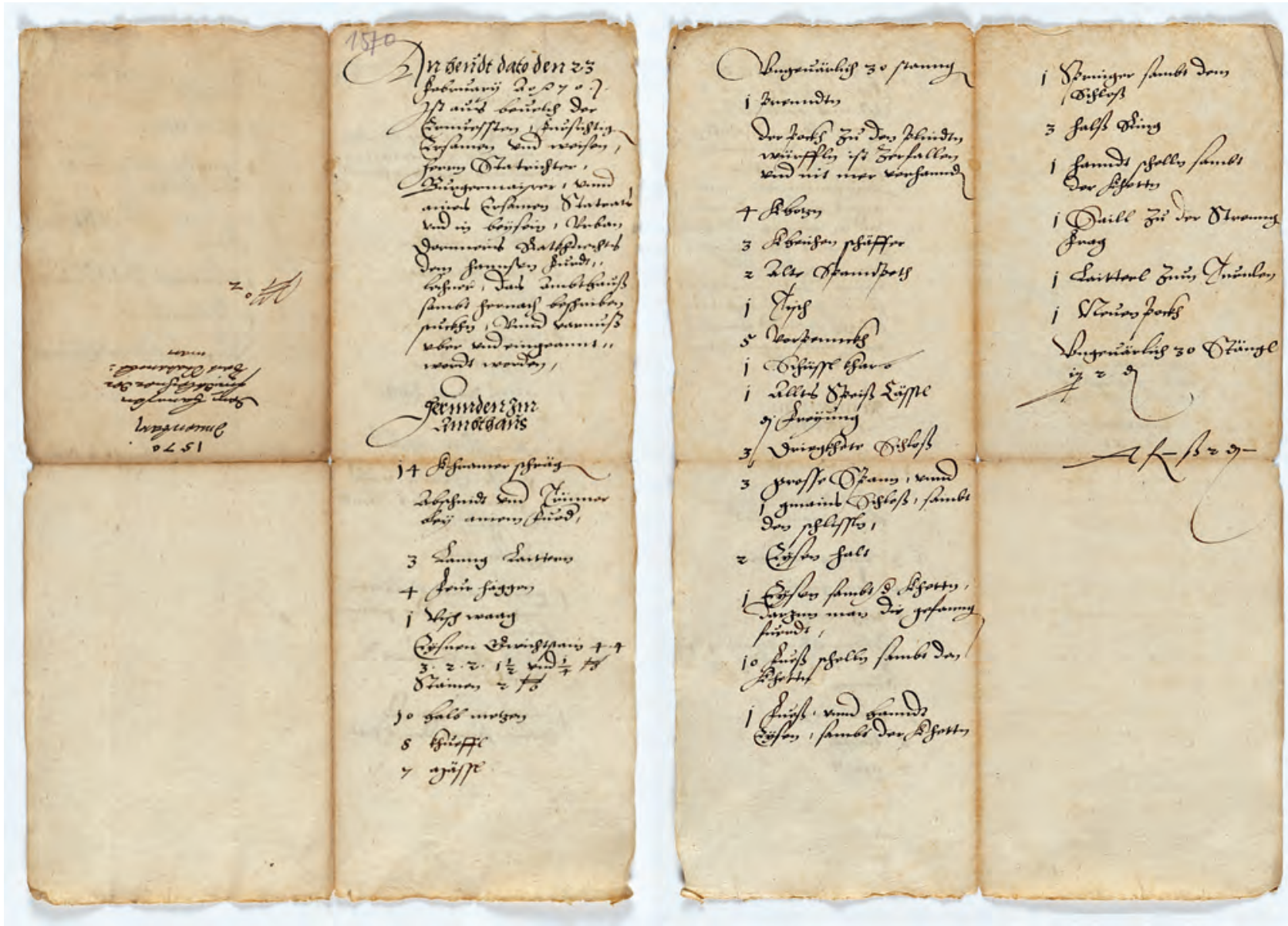

Abb. 1. Die Inventurliste des Salinenpflegerhauses vom 23. Februar 1570 führt einige Objekte auf, die später über den „Umweg“ der Zeugkammer in den Bestand des Halleiner Stadtmuseums, des heutigen Keltenmuseum Hallein gelangten (Keltenmuseum Hallein, Foto: R. Poschacher).

Dilettanten jener Jahre allenthalben in Europa angefertigt wurden (Abb. 2). Darüber hinaus hielt Seethaler die in den 1820er-Jahren geborgenen Altertümer einer „heidnischen Vorzeit“ in akkuraten, maßstabsgerechten Fundzeichnungen fest. ${ }^{12}$ So hat auch das Keltenmuseum bis heute Anteil an den Kindertagen der prähistorischen Archäologie Europas, in denen Seethaler sowohl ein Manual der Grabungstechnik für vorgeschichtliche Grabhügel als auch das von seinem Zeitgenossen Christian J. Thomsen in Kopenhagen erarbeitete „Dreiperiodenystem“ vermutlich unabhängig auf Basis lokaler Funde und antiker Ideen konzipierte. ${ }^{13}$ Dass nicht Seethaler, sondern Thomsen (und sein Kollege und Nachfolger Jens J. A. Worssae) als „Entdecker“ der Abfolge Steinzeit - Bronzezeit - Eisenzeit gilt, mag auch der mangelnden Publizität der Hallein-Salzburgischen

12 WeNDLING 2013, 56-59.

13 Seethaler $1831,68-69$.
Manuskripte geschuldet sein. ${ }^{14}$ Das Konzept an sich scheint indes, wie es Manfred K. H. Eggert mir gegenüber einmal formulierte, „damals wohl ganz allgemein im Schwange gewesen zu sein“.

\section{Frühe Forschung am Dürrnberg}

Weder zu dieser frühen Zeit noch bis weit ins 20. Jh. hinein wurde gleichwohl je an die Möglichkeit eines „Fundortmuseums“ gedacht, das dem wissenschaftlichen Stellenwert eines der bedeutendsten prähistorischen Fundorte Europas gerecht geworden wäre. Die Initiative ging zunächst vom Salzburger Museum aus, das, erst 1834 gegründet, gemeinsam mit der Bergwerksverwaltung 1857 die ersten systematischen Ausgrabungen finanzierte und organisierte. Da die erhofften Funde - erst kurz zuvor hatte Johann G. Ramsauer (1795-1874) in Hallstatt Sensationelles entdeckt ${ }^{15}$ -

14 Hansen 2001. - Eggert 2012, 29-43.

15 VON SACKEN 1868. - BARTH 1996. 


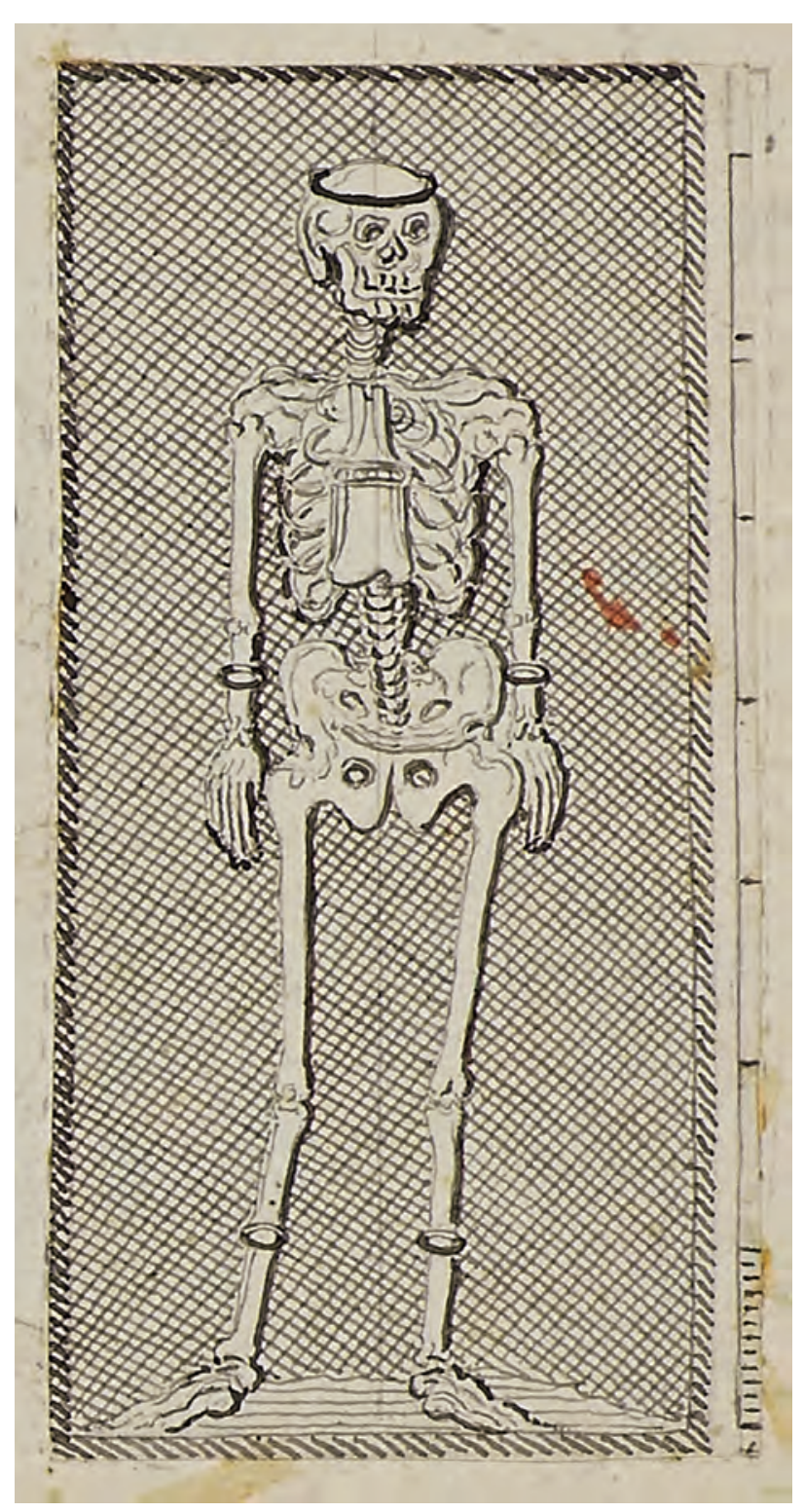

Abb. 2. Tuschezeichnung eines eisenzeitlichen Grabbefundes (heute Grab 88) vom Dürrnberg bei Hallein aus Johann A. Seethalers Manuskript „Die allerersten Celtischen und Römischen Alterthümer am Dürrnberg und zu Hallein an der Salza in Verbindung mit ihren Salinen“ von 1831 (Salzburg Museum, Inv.-Nr. 6789-49, SEETHALER 1831, Beilage).

ausblieben, profitierte das Vaterländische Museum in der Landeshauptstadt schon seit 1851 von teilweise reichen Schenkungen, die 1878 in einem größeren Konvolut an Bergwerksfunden aus den Händen der Direktion der Halleiner Saline gipfelten (Abb. 3). ${ }^{16}$

16 Wending 2016, 38. - Ramsauer selbst war auch im Halleiner Bergwerk aktiv, von dem er eine Grubenkarte und ein Modell anfertigte: WAGNER 1906b, 6.
Im selben Jahr rückten die Kelten des Dürrnbergs konkreter in den Fokus der Salzburger Öffentlichkeit, als die „Gesellschaft für Salzburger Landeskunde“ ihre Jahrestagung in Hallein abhielt. Die Archäologie, die in diesen Jahren erstmals von dem Geografen und späteren Grazer Universitätsrektor Eduard Richter (1847-1905) aufgearbeitet wurde, spielte im dortigen Stadtmuseum indes noch keine Rolle. ${ }^{17}$ Nur wenige Jahre später, 1881, wurde der Blick der (Fach-)Öffentlichkeit auch überregional erstmals auf den Dürrnberg gelenkt, als die Exkursion der in Salzburg tagenden „2. Versammlung österreichischer Anthropologen und Urgeschichtsforscher“ „die Fahrt auf den Dürrenberg bei Hallein und Einfahrt in die schon von den Kelten betriebenen Salzgruben" einschloss. ${ }^{18}$ Unter deren Teilnehmer reihten sich so illustre Gäste wie der Nestor der Anthropologie und Urgeschichte Rudolf Virchow (1821-1902), Otto Tischler (1843-1891), dem die eisenzeitliche Chronologie die Unterteilung der Hallstatt- und Latènezeit verdankt, Johannes Ranke (1836-1916), Gründer der Prähistorischen Sammlung München, oder Bertha Krupp (1831-1888), Gattin des „Kanonenkönigs“ Alfred Krupp. Funde wurden dereinst nicht im Halleiner Museum, sondern unter Tage inspiziert, wo „ein eigener Raum [...], einem unterirdischen Museum vergleichbar, noch eine weitere Anzahl von Fundstücken allerlei Art aus jener frühen Zeit"19 bewahrte. Obwohl zu dieser Gelegenheit bereits in einem Atemzug mit Hallstatt genannt, mussten sich die quantitativ und qualitativ moderaten Dürrnberger Altertümer dann aber doch den spektakulären archäologischen Erfolgen beugen, die Ramsauer im prähistorischen „Konkurrenzbetrieb“ in Oberösterreich gemacht hatte. Möchte man hiernach der „kontrafaktischen Geschichte“ unter dem Motto „was wäre, wenn... “ folgen, so mag man über die (fiktiven) Auswirkungen potentieller früher Großgrabungen spekulieren: Würde der „Hallstattzeit“ die „Dürrnbergzeit“ folgen? Der schwedische Reichsantiquar Hans Hildebrand (18421913), der die ältere Eisenzeit nach dem eponymen Fundort im Salzkammergut benannte, findet sich jedenfalls nicht auf der Teilnehmerliste des Salzburger Treffens von $1881 .^{20}$

Als sich rund ein Vierteljahrhundert später die Deutschen und Wiener anthropologischen Gesellschaften 1905 zur gemeinsamen Tagung in Salzburg trafen, führte eine der Exkursionen wiederum nach Hallein sowie auf und in den Dürrnberg. Neben den dortigen prähistorischen Abbauspuren stand in Hallein die Besichtigung der „Fürstenzimmer“

17 Richter 1879. - Richter 1880. - Richter 1881.

18 Much 1882, 2, 70-72.

19 Much 1882, 71.

20 Much 1882, 73-76. - Vgl. Penninger 1963, 6. 


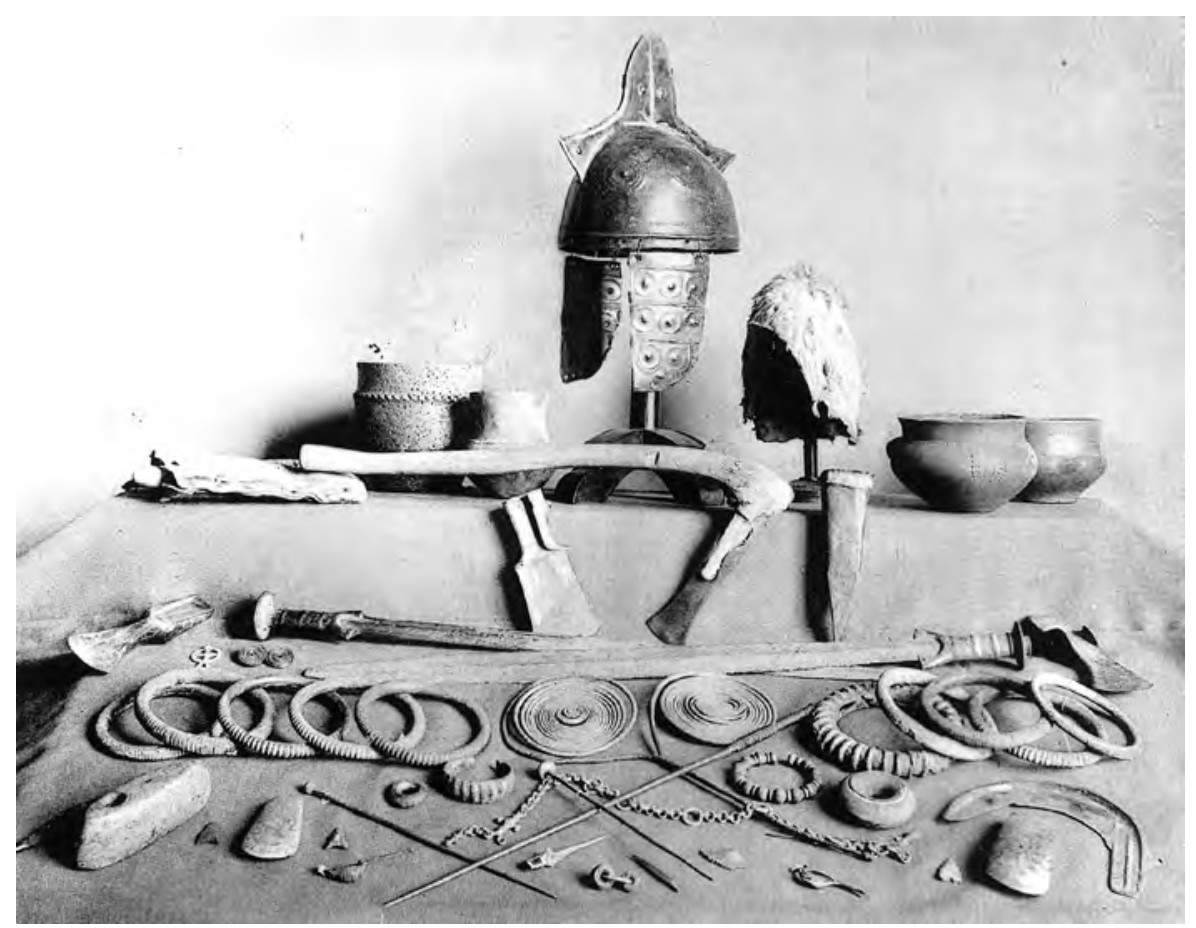

Abb. 3. Unter den prähistorischen Funden, die in einer der frühesten Bestandsfotografien des Salzburger Museums Carolino Augusteum festgehalten wurden, finden sich zahlreiche Objekte aus dem Salzbergbauzentrum auf dem Dürrnberg (Salzburg Museum, aus: SMCA 1898, Taf. 1).

im Pfleggebäude sowie erstmals auch des Museums auf dem Programm. ${ }^{21}$

Ansonsten scheint das Halleiner Museum in jenen Tagen in einem Zustand der - zumindest archäologischen Nichtbeachtung geschlummert zu haben, wohingegen Karolina „Lina“ (1890-1978) und Martin Hell (1885-1975), kongeniale Impulsgeber der Salzburger Archäologie, das keltische Bergbauzentrum in ihren archäologischen Aktionsradius aufnahmen..$^{22}$ Ihre Funde verblieben einstweilen in der Hell'schen Privatsammlung, von wo sie letztlich in das Salzburg Museum gelangten. Zwar blieb das Halleiner Museum weiterhin „fundleer“, konnte aber doch auf Betreiben des ehrenamtlichen Kustos, des Schulrats Sebastian Greiderer 1928 einen maßgeblichen Fortschritt verbuchen: Im Parterre des Rathauses wurden sieben Räume adaptiert, die neben historischen Objekten auch eine kleine urgeschichtliche Kollektion beherbergten. ${ }^{23}$ Allerdings

21 Much, Petter, Bouchal 1906, [76]. - Wagner 1906a, 5. 22 Hell 1914. - Hell 1916. - Hell 1926. - Hell 1929. - Das im Druck erschienene „Verzeichnis I der im städtischen Museum zu Hallein aufbewahrten Gegenstände. Nach dem Stande vom 1. Jänner 1914“ führt trotz des „Interesses für die örtliche kulturelle und künstlerische Vergangenheit“ keine archäologischen Objekte auf. 23 Penninger 1963, 4. bestand diese aus der Privatsammlung des Salinenarztes Karl Mittermayer, dessen Witwe sie der Pflege durch Martin Hell überantwortete. ${ }^{24}$ Das Museum selbst konnte bis 1949 nur 14 prähistorische Objekte im „Altbestand“ sein Eigen nennen, von denen ursprünglich drei - ein Hiebmesserfragment, eine Bronzegürtelkette und die als Relief ausgeführte bronzene Kopfapplike - der eisenzeitlichen Besiedlung des Dürrnbergs entstammten. ${ }^{25}$ Eine mutmaßlich metallzeitliche Geweihaxt vom Georgenberg bei Kuchl lenkte schon damals den Blick auch auf die Urgeschichte der Region (Abb. 4). ${ }^{26}$ Noch 1930 wurde das neu situierte Heimatmuseum anlässlich des 700. Jahrestags der Halleiner Stadterhebung feierlich eröffnet, so dass heuer, neben dem 50. Geburtstag des Keltenmuseums mit dem 90. Jubiläum des institutionalisierten Stadtmuseums doppelter Anlass zur Freude besteht. ${ }^{27}$

Schon vorher hatte der Dürrnberg neuerlich die Fachkreise in seinen Bann gezogen, die sich 1926 zur gemeinsamen Tagung der „Deutschen Anthropologischen

24 Penninger 1972, 28.

25 Inventarbuch Penninger „Altbestand des Museums“.

26 Inv.-Nr. AR_1970_0139. - Hell 1931. - Hell 1934. - PeNNINGER 1972, 28.

27 Volksfreund 1930, 3-4. - Penninger 1963, 4. 

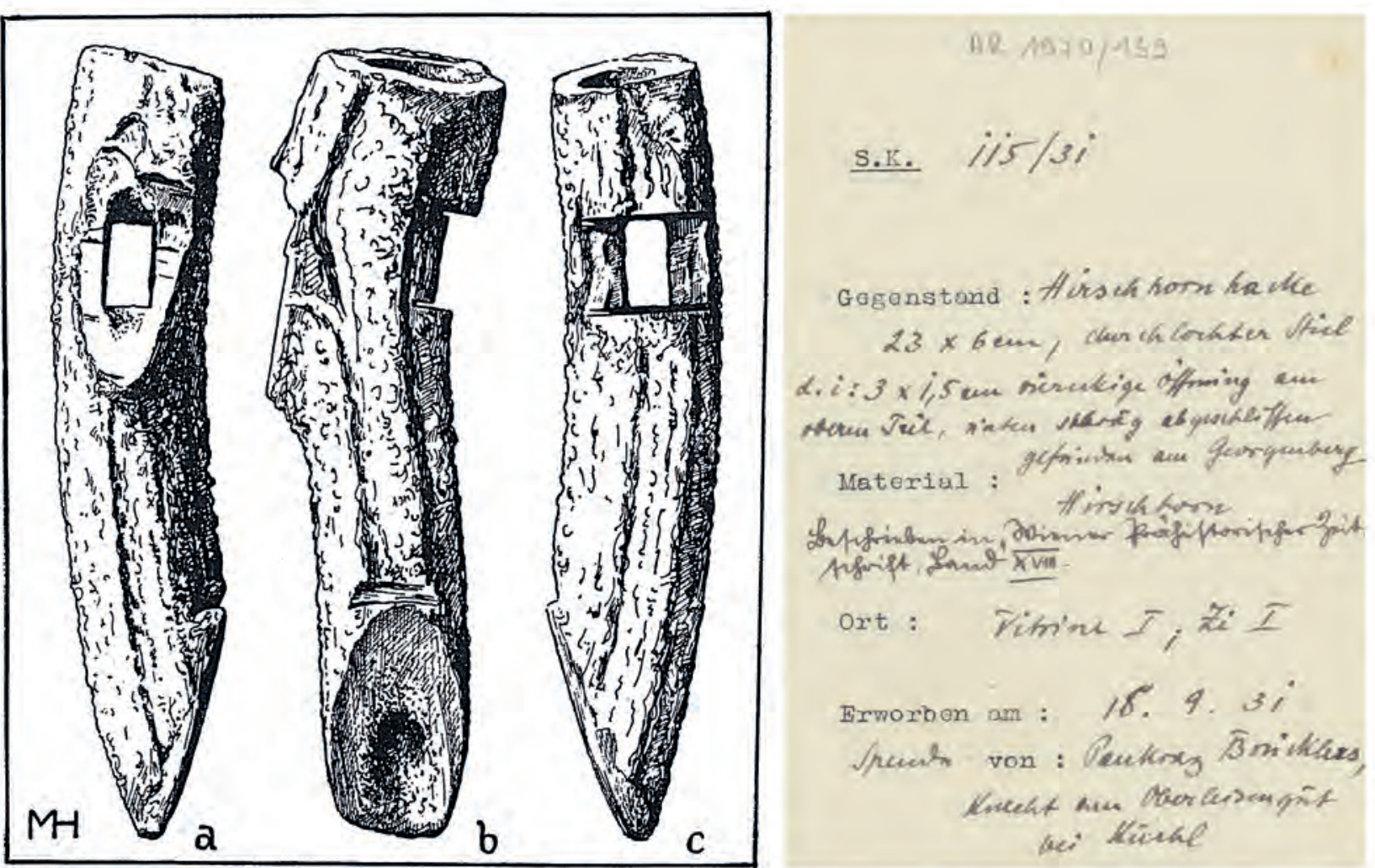

Abb. 1. Hirschhornaxt vom Georgenberg bei Kuchl, Salzburg $(1 / 3$ nat. Gr.).

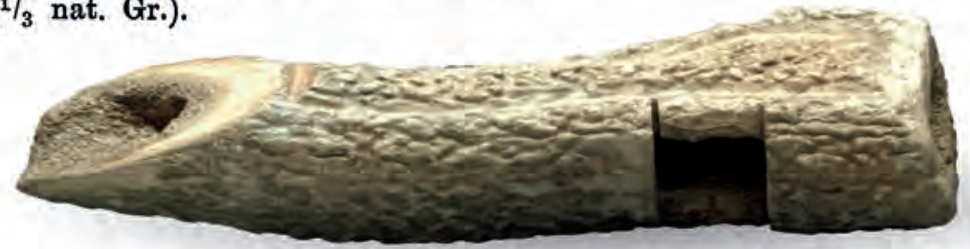

Abb. 4. Eine mutmaßlich metallzeitliche Geweihaxt vom Georgenberg bei Kuchl gehört zum ältesten Bestand der archäologischen Sammlung des Keltenmuseum Hallein (Keltenmuseum Hallein, Inv.-Nr. AR_1970_0139; Zeichnung: Hell 1931, 153 und Abb. 1; Foto: D. Böckmann).

Gesellschaft“ und der „Anthropologischen Gesellschaft in Wien“ in Salzburg einfanden. In Vortrag und Exkursion erörterte Hell die Situation und präsentierte im Dürrnberger Schulhaus die neuesten Funde aus der Privatsammlung des Oberlehrers Georg Suppin (1883-1967). ${ }^{28}$ Die Teilnehmerliste liest sich wie ein who is who der damaligen Urgeschichtsforschung und nennt - in kleiner Auswahl - Robert Beltz, Ferdinand Birkner, Karl H. Jacob-Friesen, ${ }^{29}$ Otto Kunkel, Georg Kyrle, Rudolf Much, Carl Rademacher, Hans Reinerth, Robert R. Schmidt, Georg Schwantes oder

28 Hell 1927a. - Hell 1927b. - Vgl. Wendling 2013, 63. 29 Hier offenbar missverständlich als „Friesen, Jakob Dr., Hannover“ aufgeführt.
Ernst Sprockhoff. Auf Olivier Klose (1860-1933), Kustos der archäologischen Sammlung des Salzburger $\mathrm{Mu}$ seums, machte das archäologische Potential des Halleiner Salzreviers offenbar dermaßen großen Eindruck, dass er sich, nicht ohne Missmut zu erregen, nun auch in der dortigen Feldforschung engagieren wollte. ${ }^{30}$ Er deckte in wenigen Grabungskampagnen der Jahre 1926-1932 nicht weniger als 16 Gräber bzw. Grabhügel der Hallstatt- und Latènezeit auf. ${ }^{31}$ Unter ihnen befand sich auch das nach heutiger Zählung mit der Ziffer 112 belegte Wagengrab, dessen antiker

30 Zur Grabungsgeschichte des Dürrnbergs: MoosLeitner 1977. Penninger 1980. - Wendling 2013.

31 Penninger 1972, 26-27. 

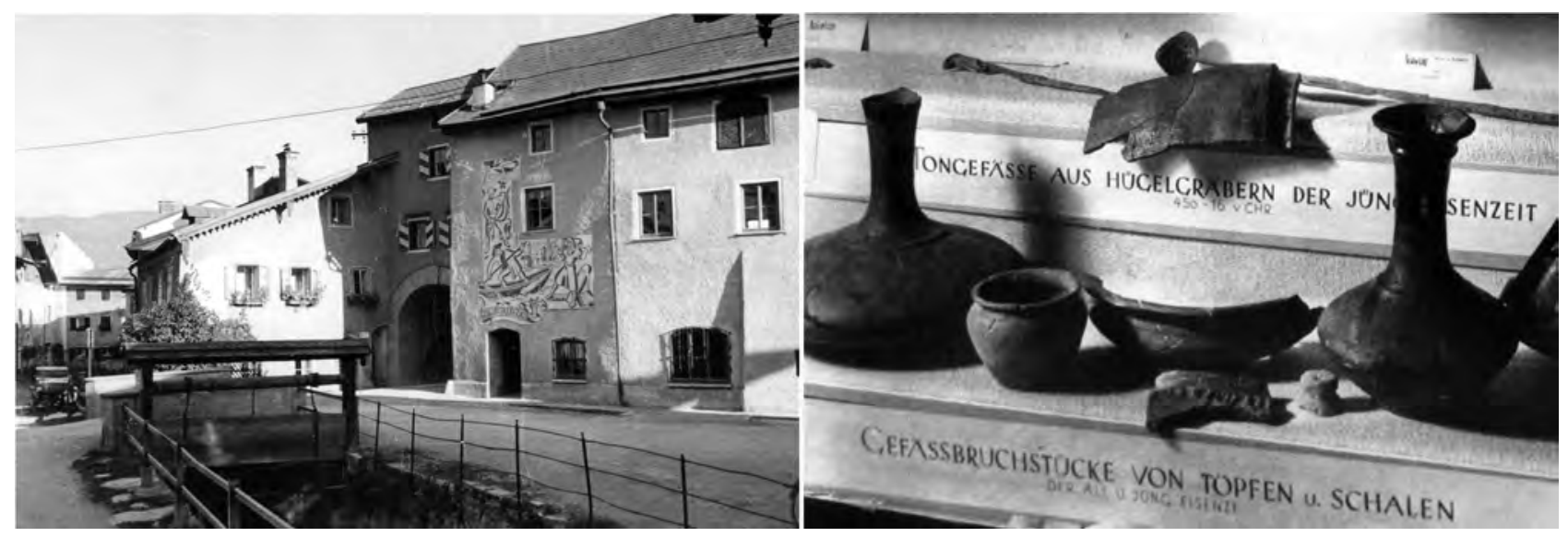

Abb. 5. Das „Untere Griestor“ beherbergte seit 1952 das Halleiner Stadtmuseum (Fotos: Keltenmuseum Hallein, Stadtarchiv Hallein, Inv.-Nr. FO_2013_0120, FO_2015_0074).

Beraubung die berühmte Dürrnberger Schnabelkanne entgangen war. Klose integrierte die Kanne, die schon bald für eine der Ikonen der „frühkeltischen Kunst“ galt ${ }^{32}$ und als Sujet den Titel dieser Zeitschrift ziert, rechtmäßig in die Kollektion des Salzburger Museums, konnte den Ruhm des Finders allerdings nicht auskosten: Die Erstvorlage erfolgte posthum $1934 .{ }^{33}$

\section{Heimatmuseum und Keltenmuseum - die Ära Penninger}

Schon vor dem 2. Weltkrieg wurden die Museumsräume aufgelöst und das Inventar nach intensivem Briefwechsel zwischen Stadtgemeinde Hallein, dem Landesstatthalter Albert Reitter (1896-1962) und der zuständigen „Zentralstelle für Denkmalschutz“ in Wien ausgelagert. Nach dem Krieg reaktivierte man das Museumprojekt erst 1949 durch die Initiative Ernst Penningers, des Leiters der Halleiner Salzbergbahn und neugewählten Kulturreferenten der Stadt. ${ }^{34}$ Schon 1952 gelang es ihm, der in der Zwischenzeit auch durch Übernahme aller Dürrnberger Neufunde die Sammlung hatte anwachsen lassen, im „Unteren Griestor“, einem Relikt der mittelalterlichen Halleiner Stadtbefestigung, eine neue Dauerausstellung zu etablieren und $\mathrm{zu}$ eröffnen (Abb. 5). Vierzehn Schau- und zwei Depoträume beherbergten das gesamte historische Repertoire der über 100-jährigen Sammlungsaktivität: Sakrale und profane Kunst und Volkskultur, Gitarre und Autografen aus dem Besitz Franz-Xaver Grubers, des Komponisten des StilleNacht-Liedes, die sogenannte Grubermühl-Krippe und

32 Jacobsthal 1935. - Jacobsthal 1944, Nr. 382 mit Taf. 184-186. 33 Klose 1934. - Zu Grab 112 zuletzt: Tiefengraber, WiltschKeSchrot'ta 2014, 71-79. - Zur Schnabelkanne: Moosleitner 1985. - Wendling 2018a.

34 Penninger 1972, 29. die mit alter Gerätschaft ausgestattete Turmküche. In einem Raum des zweiten Stocks wurde die urgeschichtliche Sammlung präsentiert, für die der Platz freilich durch die seit 1951 von Penninger vervielfachte Grabungs- und Sammlungsintensität im Grunde bereits zur Eröffnung kaum mehr ausreichte. ${ }^{35}$ Dem zu diesem Anlass mit großem Enthusiasmus gegründeten „Verein Heimatmuseum Hallein“ war trotz der von Martin Hell hervorgehobenen „internationalen Bedeutung des Halleiner Museums“ kein nachhaltiger Erfolg beschieden. ${ }^{36}$

Einen der maßgeblichen Zugewinne jener Jahre stellt das Inventar des 1959 entdeckten „Fürstengrabes“ 44\#2 dar, das gemeinsam mit dem aus dem darüber liegenden Grab 44\#1 geborgenen Goldschiffchen bis heute eines der exquisitesten Ensembles eisenzeitlicher Prunkausstattungen Mitteleuropas darstellt. ${ }^{37}$ Das Wagengrab eines frühlatènezeitlichen Kriegers im vollen Waffenornat stellt durch die Qualität und Variabilität der Objektkategorien den kosmopolitischen Charakter des nordalpinen Salzzentrums exemplarisch zur Schau (Abb. 6): Das einheimische Schmuck- und Waffenrepertoire weist seinen Träger als Angehörigen der obersten Gesellschaftsklasse aus, der dem Wagenfahrer aus dem Schnabelkannen-Grab 112 gleichkam. Die Kontakte jener frühkeltischen Elite reichten nach Ausweis des Bernsteins und einer griechischen Keramikschale von der Ostsee über Etrurien und die Adria bis nach Athen. Eine als Amulett getragene Kaurimuschel könnte gar aus dem Indischen Ozean stammen. ${ }^{38}$

\footnotetext{
35 Penninger 1963, 5.

36 Tennengauer Zeitung 1951, 2.

37 Penninger 1960a. - Penninger 1960b. - Penninger 1972, 76-80. 38 Zeller 2002. - Zeller 2003a. - Wendling in Vorb.
} 


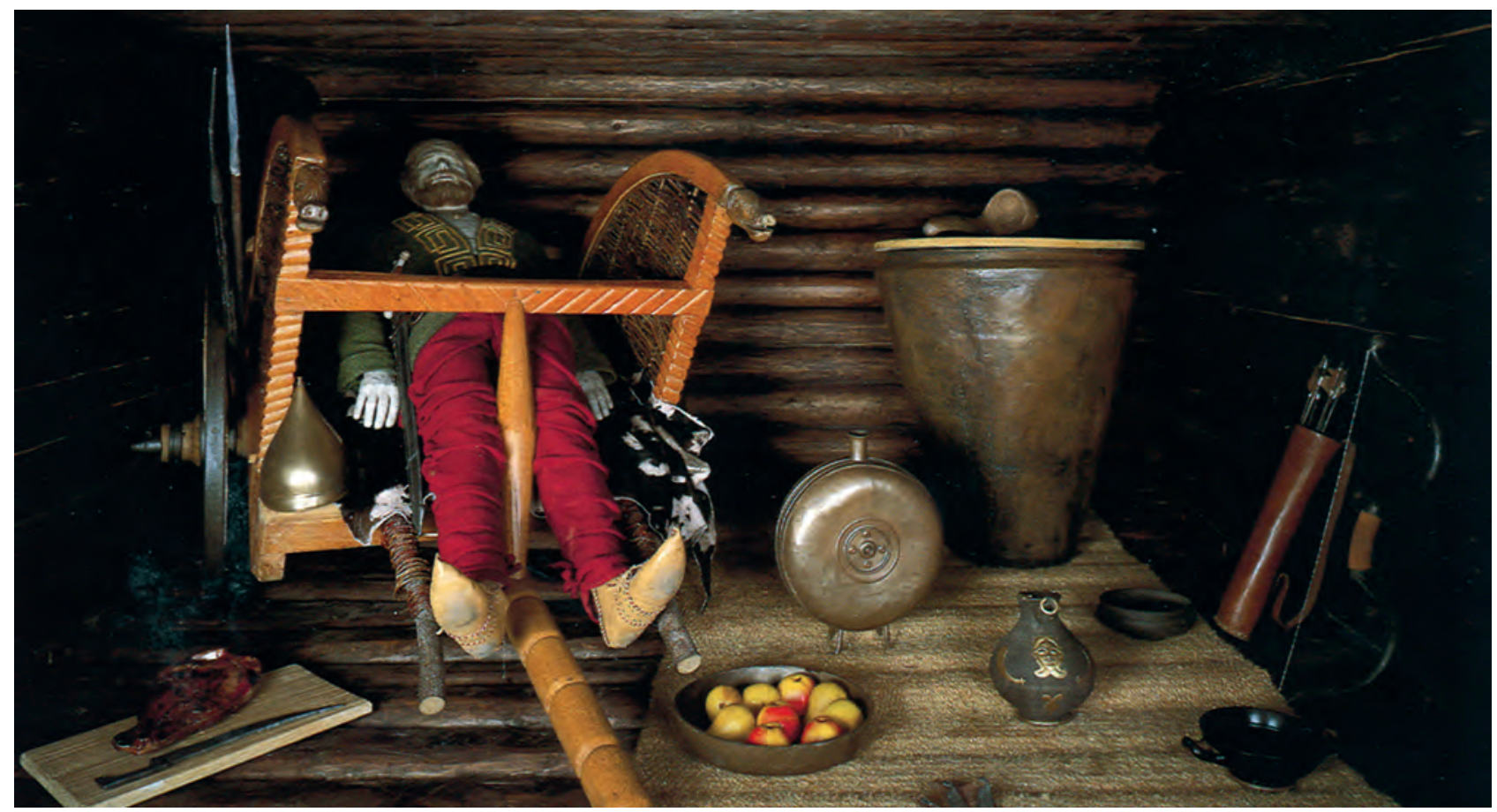

Abb. 6. Rekonstruktion der Ausstattung der Grabkammer des frühlatènezeitlichen „Fürstengrabes“ 44\#2, die 1980 für die Landesausstellung „Die Kelten in Mitteleuropa“ angefertigt wurde (Foto: Keltenmuseum Hallein).

Der spektakuläre Fundkomplex sollte unmittelbar der Öffentlichkeit präsentiert werden, wofür die Räumlichkeiten im Halleiner Stadtmuseum jedoch keinen Platz boten. Als 30. Sonderausstellung des Salzburger Museums Carolino Augusteum „Kunst der Kelten in Salzburg“ wurden die Funde schließlich von Juni bis Oktober 1960 in einer Kooperation beider Häuser im Museumspavillon des Salzburger Mirabellgartens gezeigt (Abb. 7). ${ }^{39}$ Ergänzt wurden sie durch die erst kurz zuvor von der Restaurierung am Römisch-Germanischen Zentralmuseum Mainz zurückgekehrte Schnabelkanne vom Dürrnberg, deren Umzeichnung als Ausstellungssujet diente (Abb. 8). Ein vom Direktor des Salzburger Museums Kurt Willvonseder (1903-1968) verfasstes Begleitheft zur Sonderausstellung erfuhr aufgrund der großen Nachfrage schon im selben Jahr eine zweite Auflage. ${ }^{40} \mathrm{Im}$ Anschluss an die Salzburger Schau wurde die Ausstellung während eines Monats, ergänzt durch Objekte aus Neolithikum und Bronzezeit unter dem Titel „4000 Jahre Dürrnberg" schließlich doch noch in Hallein, im dortigen Gewerkschaftsheim gezeigt. Der Erfolg der beiden archäologischen Ausstellungen ließ aufhorchen: Über $12.000 \mathrm{Be}-$ sucherinnen und Besucher an beiden Standorten und eine

39 WillvonsEDER 1960b, 169-170.

40 Willvonseder 1960a.

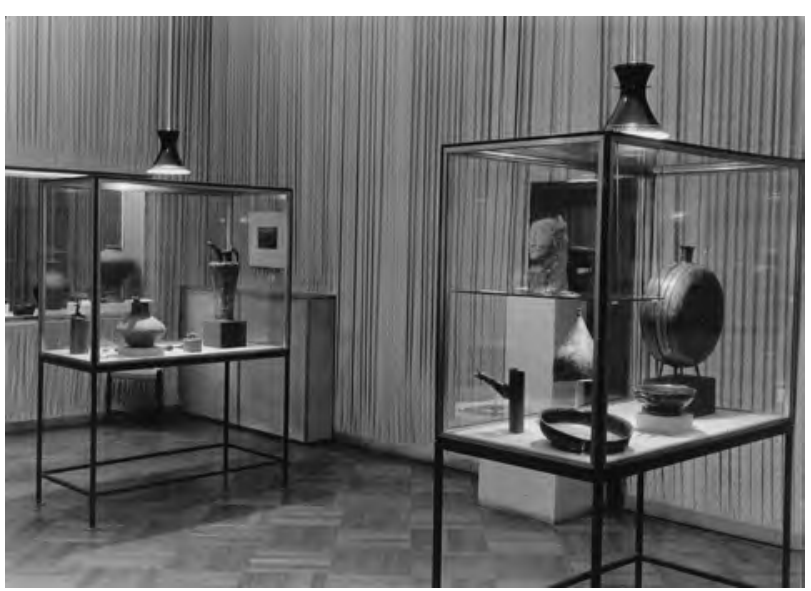

Abb. 7. Blick in die Ausstellung „Kunst der Kelten in Salzburg“, in der 1960 unter anderem die Dürrnberger Schnabelkanne sowie Objekte aus dem seinerzeit kürzlich entdeckten „Fürstengrab“ $44 \# 2$ präsentiert wurden (Foto: Salzburg Museum, Archiv FB Archäologie).

internationale Medienberichterstattung bescherten der Dürrnberger Archäologie trotz der recht kurzen Laufzeit eine immense Aufmerksamkeit. ${ }^{41}$ Hierzu mag unter anderem beigetragen haben, dass die Sonderausstellung schon vor

41 Willvonseder 1960b, 170. 


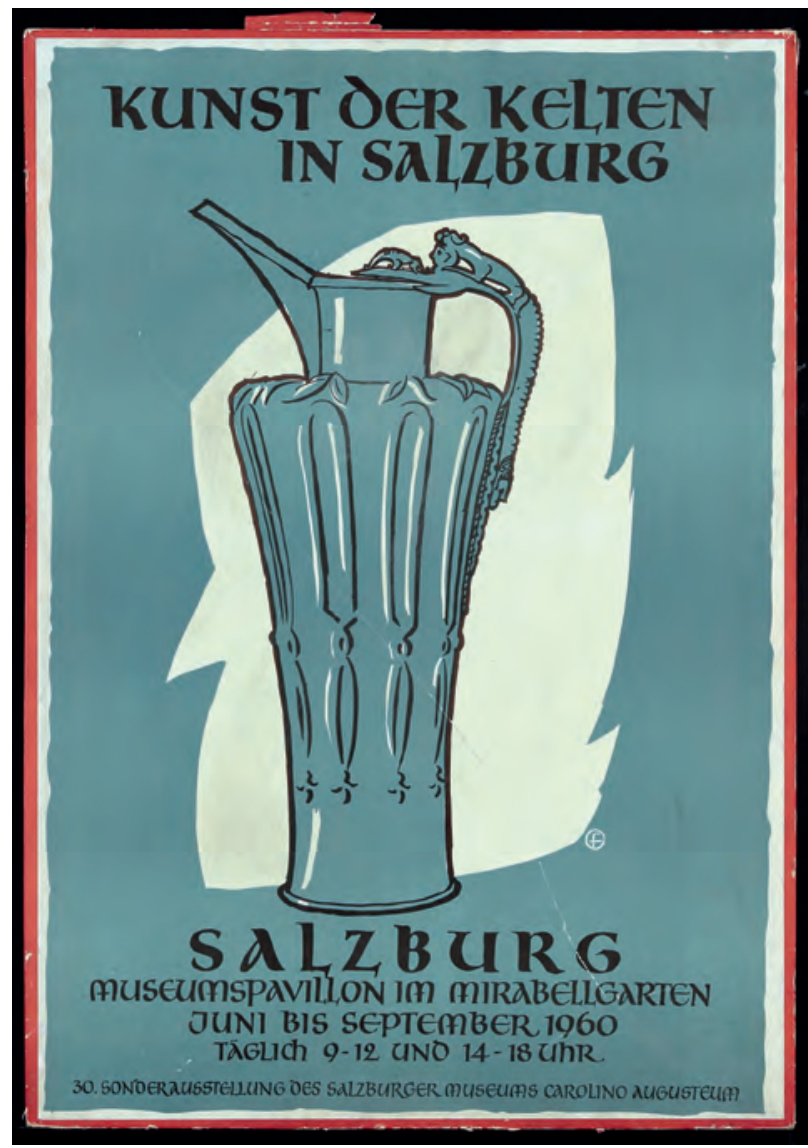

Abb. 8. Das Plakat der 1960 in Salzburg und Hallein gezeigten Ausstellung „Kunst der Kelten in Salzburg“ ziert eine Grafik der Dürrnberger Schnabelkanne (Salzburg Museum, Inv.-Nr. BIB PLA 12040).

der Eröffnung für die Jahrestagung der „Österreichischen Arbeitsgemeinschaft für Ur- und Frühgeschichte“ Anlass gab, die unter dem Schlagwort „Keltenproblem“ vom 26.-29. Mai 1960 mit über 110 Teilnehmern im „Haus Rief“ bei Hallein stattfand. ${ }^{42}$ Neben der Vorschau-Inspektion der Dürrnberger Funde, dem Empfang der Tagungsteilnehmerinnen und -teilnehmer durch Landeshauptmann und Salzburger Bürgermeister und diversen Referaten und Abendvorträgen wurde die Situation auf und im Dürrnberg auf einer Exkursion in Augenschein genommen.

Der hiesige Erfolg der Ausstellung schlug sich auch erstmals in einer internationalen Präsenz nieder, bei der die Funde 1963 im Rahmen eines Kulturaustausches im niederländischen Haarlem gezeigt wurden. Dass hierbei die romanische Skulptur eines Männerkopfes mit Brünne von der Festung Hohensalzburg die „Keltische Kunst“ repräsentierte, sei nachzusehen (Abb. 9).

42 KRENN 1961, 32-33.

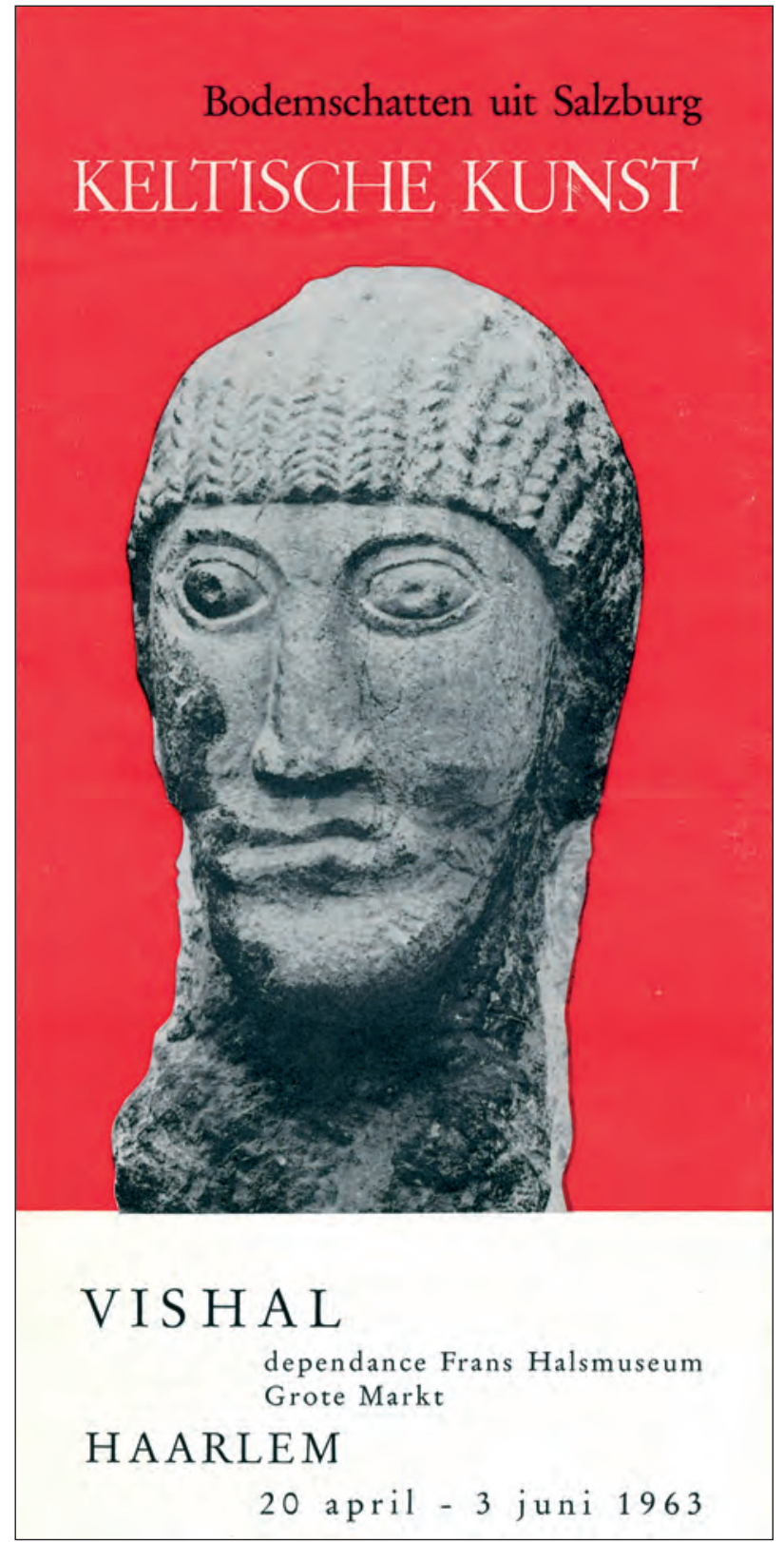

Abb. 9. Werbeprospekt für die 1963 im niederländischen Haarlem gezeigte Ausstellung „Keltische Kunst. Bodemschatten uit Salzburg"(Foto: Keltenmuseum Hallein).

Nicht nur aus den Erfahrungen der Sonderausstellung beklagte der ehrenamtliche Kustos Penninger stets die begrenzten räumlichen Möglichkeiten der kontinuierlich zunehmenden Bestände. ${ }^{43}$ Seinem Bemühen ist es zu verdanken, dass die Stadtgemeinde 1967 das Pflegamtsgebäude erwarb und bis zum Jahr 1970 für einen regulären Museumsbetrieb ertüchtigte. ${ }^{44}$ Am 17. Oktober 1970 wurde

43 Penninger 1963, 5.

44 Penninger 1972, 29. 

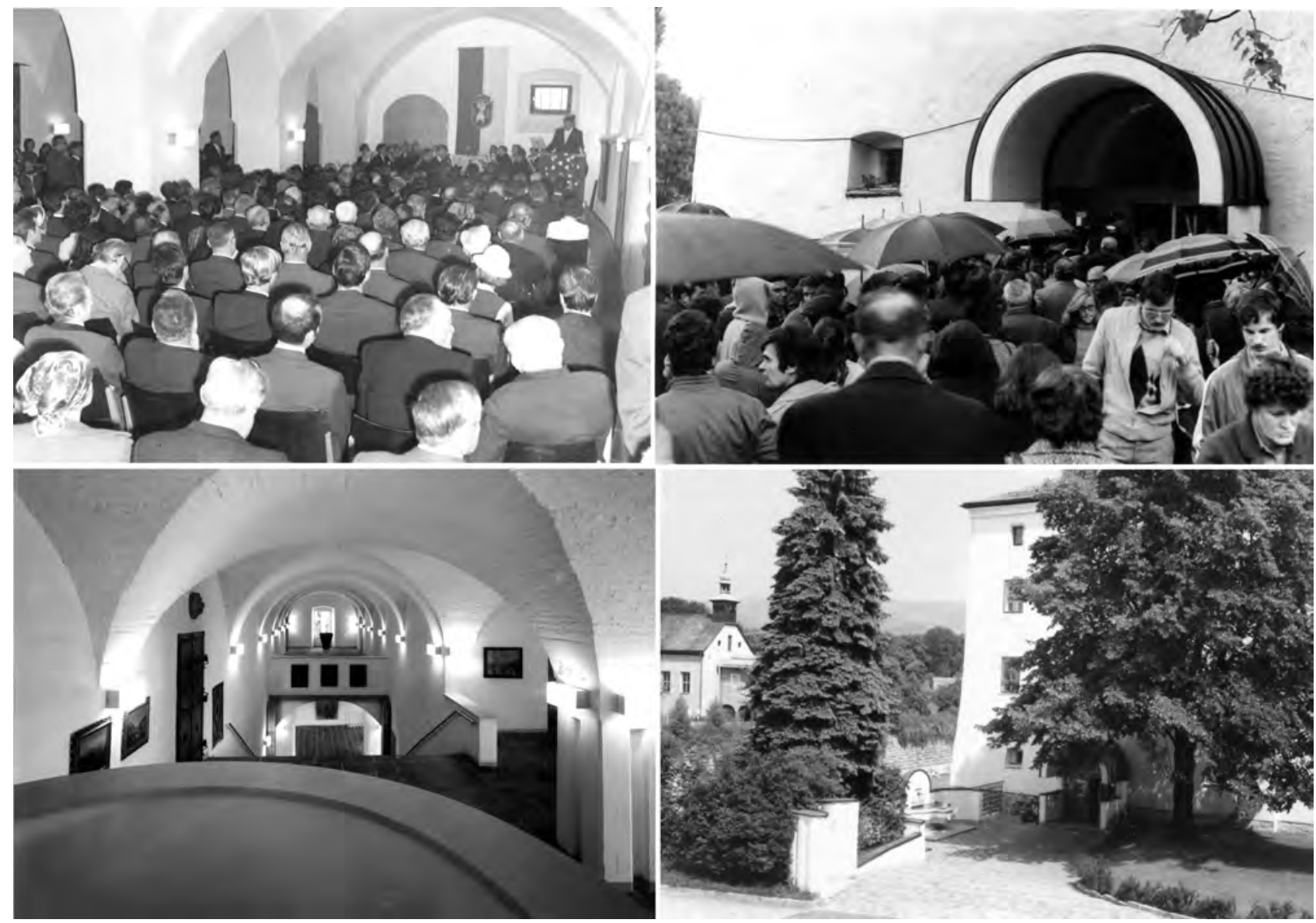

Abb. 10. Impressionen der Eröffnung des Keltenmuseum Hallein am 17. Oktober 1970. - Oben links: Eröffnungsrede E. Penningers. - Oben rechts: Besucherandrang. - Unten links: Ausstellungsbereich im 1. OG. - Unten rechts: Eingangsbereich an der Nordfront des Pfleggebäudes (Fotos: Keltenmuseum Hallein, Stadtarchiv Hallein, Inv.-Nr. FO_2015_417a.427.439a.443c).

die Einrichtung als „Keltenmuseum Hallein“ - sicherlich nicht ohne Grund mit einem deutlichen archäologischen Schwerpunkt im Namen - der Öffentlichkeit durch den Bundesminister für Unterricht und Kunst Leopold Gratz (1929-2006) und Bürgermeister und Landtagspräsidenten Josef Brandauer (1921-1988) übergeben (Abb. 10). Die Eröffnung in der neu eingerichteten „Festhalle“ im Erdgeschoß der „Pfleg“ erzeugte ein enormes Medienecho: Als „Halleiner Schatzhaus“ gefeiert, „lebt man aufgrund des Keltenmuseums wieder lieber in Hallein. "45 Auch in Fachkreisen, die zahlreich bei der zeitgleich stattfindenden Jahrestagung der „Österreichischen Arbeitsgemeinschaft für Ur- und Frühgeschichte" vertreten waren, fand die Ausstellung Zuspruch. Martin Hell notierte in seinem Archäologischen Tagebuch anlässlich der Eröffnung: „Penninger hat das

45 Halleiner Zeitung 1970a. - Halleiner Zeitung 1970b. SALzburger NaChrichten 1970.
Museum vorzüglich gestaltet. Die Urgeschichte ist einzigartig mit Hallstatt- und Latènezeit vertreten. “ ${ }^{46}$

Neben der Direktion des Keltenmuseums, die Penninger bis 1989 innehatte, wirkte er von 1976 bis 1985 als Landesarchäologe und konnte aufgrund seiner eigenen, seit 1949 auf dem Dürrnberg durchgeführten Grabungen in den Anfangstagen des Keltenmuseums laut Inventarbuch bereits auf einen Bestand von rund 1900 archäologischen Objekten zurückgreifen. Eine Auswahl wurde in sechs der Urgeschichte des Dürnbergs gewidmeten Räumen präsentiert, die im damals baulich auf „die Pfleg“ beschränkten Rahmen noch den 17 Räumen der Stadtgeschichte hintanstanden. ${ }^{47}$ Durch die archäologischen Aktivitäten entwickelte sich das Keltenmuseum allerdings konsequent zu einem veritablen Fundortmuseum, das durch die enge

46 Hell 1970.

47 Penninger 1976, 44-46. 
räumliche Verwobenheit von archäologischer Fundstätte und musealer Präsentation dem internationalen Fach- und Laienpublikum gleichermaßen unmittelbare Ein- und Ausblicke in die Archäologie der eisenzeitlichen Salzmetropole bot.

Der Institutionalisierung des Keltenmuseums ist es unter anderem zu verdanken, dass die durch den Bau der Landesstraße 256 auf weiten Strecken akut gefährdete archäologische Substanz des Dürrnbergs gesichert und dokumentiert werden konnte. ${ }^{48}$ Die im gemeinsamen Bemühen des Keltenmuseums, des Salzburger Museums C. A., der Landesarchäologie und des Bundesdenkmalamtes ergrabenen, vormals nicht oder nur teilweise bekannten $\mathrm{Ne}$ kropolen entlang der modernen Trasse bedeuteten einen erheblichen Zuwachs an gut dokumentierten Gräbern und Siedlungsstellen sowie vielfältiges Fundmaterial, das gesamthaft in das Depot des Keltenmuseums eingegliedert wurde.

Während jener Grabungen, besonders aber der bis heute geradezu legendären Ruf genießenden Landesausstellung „Die Kelten in Mitteleuropa. Kultur - Kunst - Wirtschaft“ tat sich Kurt W. Zeller (1945-2009) als in Hallein und der Region tief verwurzelter Enthusiast hervor, der in den kommenden Jahrzehnten den Weg des Museums und der Forschung auf dem Dürnberg bestimmen sollte (Abb. 11). Letztere prägte in den Jahren vor und nach den „linearen“ Notgrabungen zudem maßgeblich der Münchener Prähistoriker Ludwig Pauli (1944-1994), der neben Penninger und dem späteren Landesarchäologen Fritz Moosleitner die Vorlage der bis Ende der 1970er-Jahre ergrabenen Bestattungen voranbrachte. ${ }^{49}$ Besonders die in Band III der Publikationssequenz vorgenommene „Auswertung der Grabfunde“ setzt bis heute in formenkundlichem Weitblick, präziser intellektueller Argumentation und stilistischer Eleganz Maßstäbe. ${ }^{50}$ Sein „Versuch einer historischen Auswertung“ ging weit über die sozioökonomische Situation am Dürrnberg hinaus und nahm ethnische Fragen ebenso wie das große Panorama der „Entstehung und Ausbreitung der Latènekultur" ins Visier. Sein Werk, in dem der Dürrnberg eine ganz zentrale Position innehatte, bildete eine wichtige Grundlage für die öffentlichkeitswirksame Vermittlung und wissenschaftliche Rezeption der Salzmetropole als einem der bedeutendsten Fundorte der mitteleuropäischen Eisenzeit. ${ }^{51}$ In Anerkennung dieser Leistungen erhielt er 1980 das „Silberne Ehrenzeichen des Landes Salzburg“ “. 52

\footnotetext{
48 Zeller 1980.

49 Penninger 1972. - Moosleitner, Pauli, Penninger 1974.

50 Pauli 1978. - Kossack 1995.

51 So bereits Penninger 1963, 5. - Maier 1974.

52 Kossack 1995, 14.
}

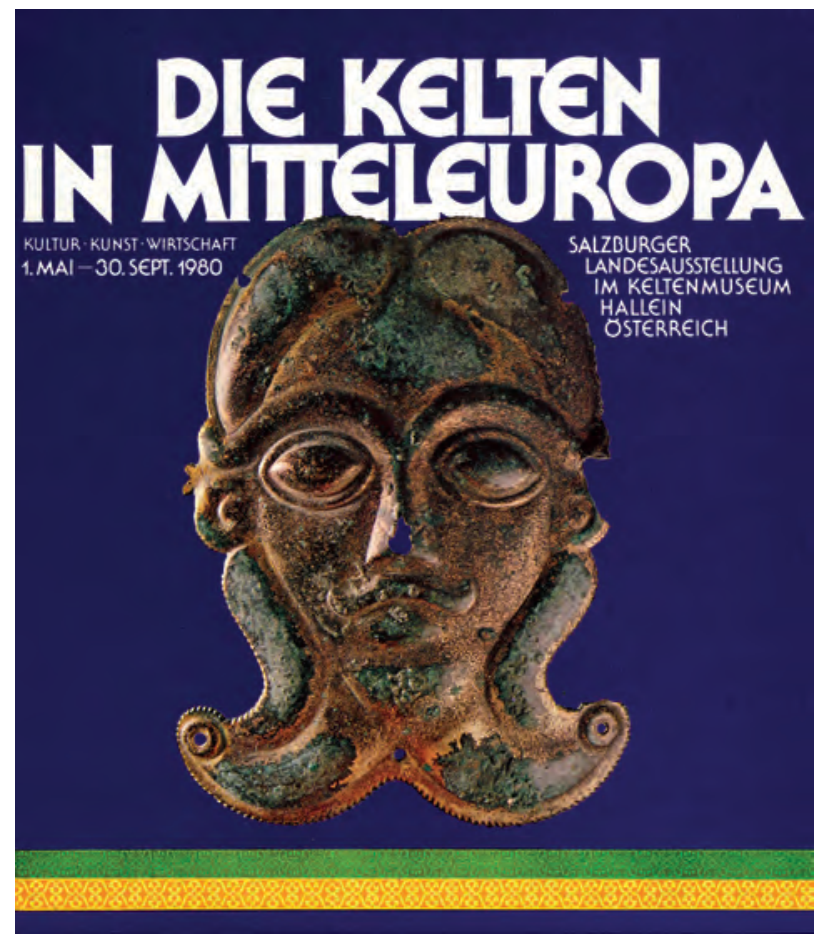

Abb. 11. Die Salzburger Landesausstellung „Die Kelten in Mitteleuropa" von 1980 gilt als Ursprung und Vorbild der späteren internationalen Großausstellungen zur Kultur der Kelten (Foto: Keltenmuseum Hallein).

Mit über 350.000 Besucherinnen und Besuchern bot die Landesausstellung 1980, im zehnten Jahr des Bestehens des Keltenmuseums, eine umfassende Schau zur prähistorischen keltischen Kultur Mitteleuropas, die europaweit Beachtung fand und den großen Keltenausstellungen der kommenden Dekaden, etwa in Venedig 1991, Rosenheim 1993, Frankfurt 2002 oder Stuttgart 2012 Inspiration war. Zeller war seinerzeit auch mit der Organisation der „Freilichtschau“ im Ortszentrum von Bad Dürrnberg befasst, die in rekonstruierten Hausbauten und einer dem damaligen Forschungsstand entsprechenden Inneneinrichtung das Leben der Dürrnberger Kelten vor 2500 Jahren schilderte. Einen spektakulären Blick in Grabsitten und Jenseitsvorstellungen jener Zeit bot zudem die Rekonstruktion des „Fürstengrabes“ 44\#2 (Abb. 6). 1996 wurde das „keltische Gehöft" den Salzwelten Hallein überantwortet, 1998 in einer aufwendigen Aktion per Hubschrauber in das Vorfeld des Besucherbergwerks versetzt und um einige neue Bauten ergänzt..$^{53} \mathrm{Da}$ der Zahn der Zeit auch an jenen modernen Installationen genagt hatte, entschlossen sich die Salzwelten 2013, das Keltendorf SALINA zu reaktivieren und als

53 Zeller 1997, 12-13. 


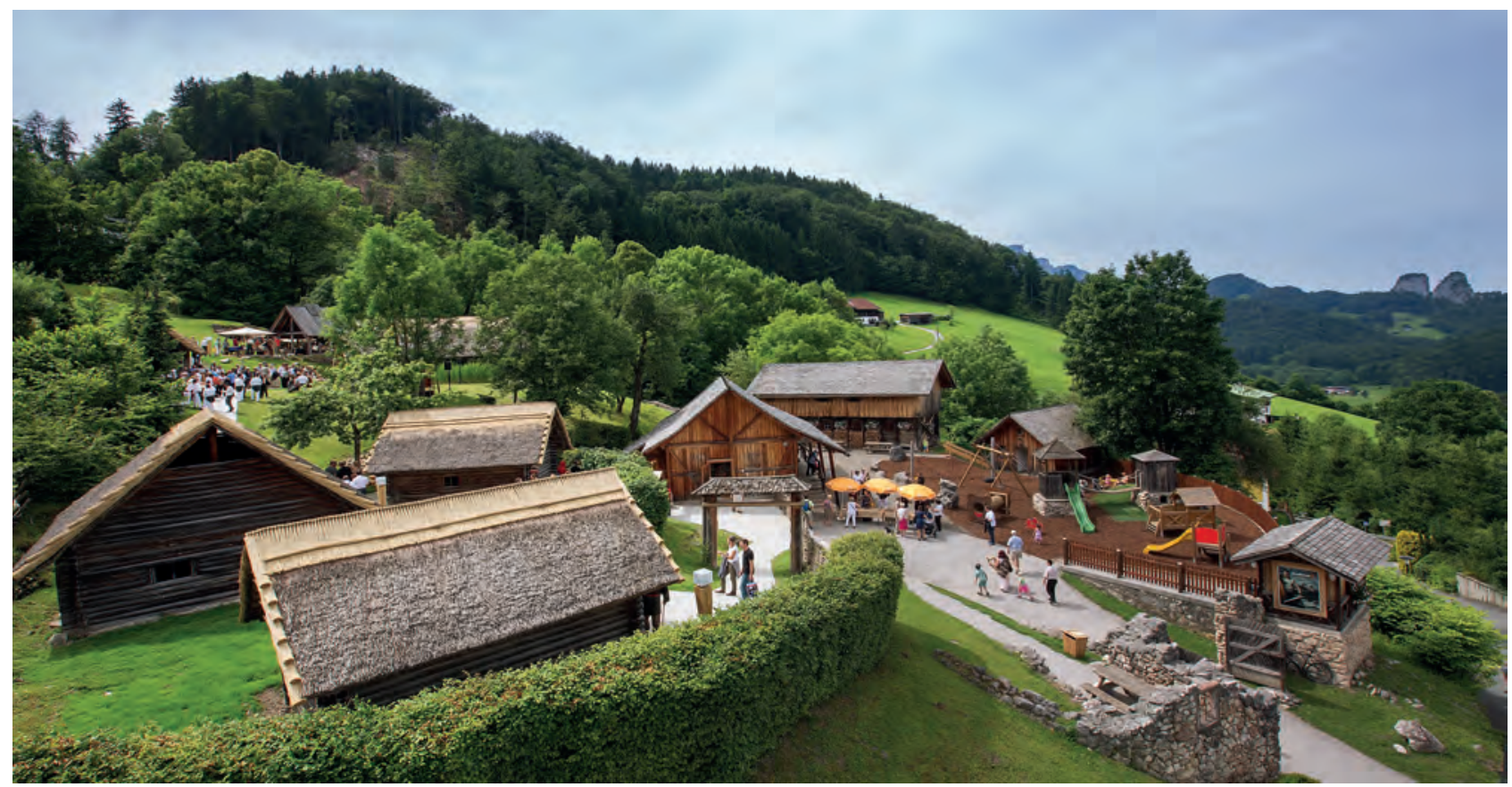

Abb. 12. Der 2014 wiedereröffnete Freilichtbereich „Keltendorf Salina“ erfüllt auch als touristisch konzipiertes Besucherzentrum der Salzwelten Hallein wissenschaftliche Standards (Foto: Salzwelten Salzburg).

attraktiven Teil des Besucherbergwerks zu modernisieren. Mit wissenschaftlicher Expertise der Dürrnbergforschung am Keltenmuseum Hallein wurde so ein modernsten museumspädagogischen Anforderungen gerecht werdendes Besucherzentrum geschaffen, das seit seiner Eröffnung im Jahr 2015 mit bunten Lebensbildern die Realität der keltischen Dürrnberger Bergleute erzählt (Abb. 12). ${ }^{54}$ Im Eingangsbereich vermitteln rekonstruierte Grabungssituationen die archäologische und anthropologische Arbeitsweise vom Befund und Fund über die wissenschaftliche Analyse zur grafischen Rekonstruktion.

\section{Keltenmuseum und Österreichisches Forschungszentrum Dürrnberg}

Kurt W. Zeller wurde 1985 zunächst die Leitung des neu gegründeten „Österreichischen Forschungszentrums Dürrnberg“ (ÖFD) übertragen. Das ÖFD sollte den besonderen Anforderungen des Dürrnbergs in den Bereichen archäologischer Administration, Denkmalpflege und Forschung gerecht werden. Hierzu brachten die Stadtgemeinde Hallein, das Land Salzburg, die Republik Österreich und die Salinen Austria AG als Betreiber des lokalen Bergwerksbetriebes Mittel auf. ${ }^{55}$ Von den Aktivitäten des

54 Wendling 2015a.

55 Zeller 1990. - ZelLer 1997.

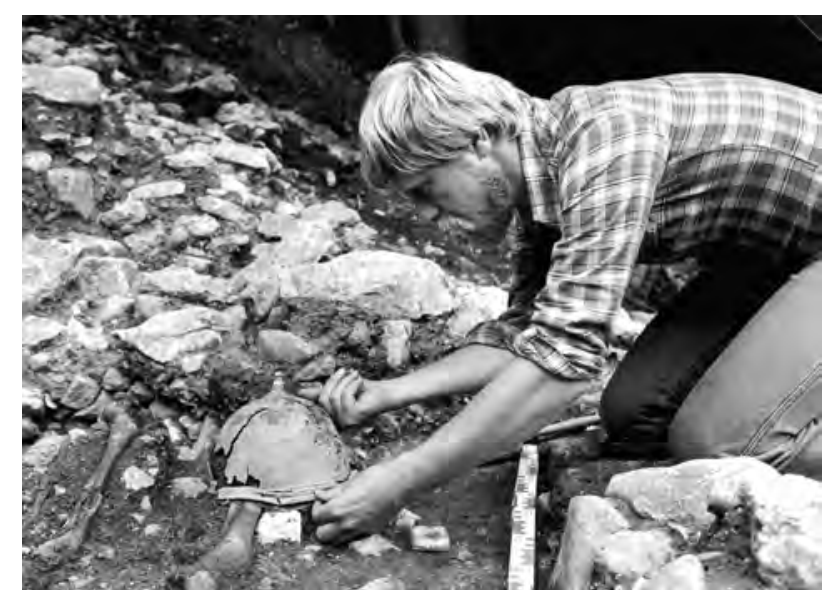

Abb. 13. Kurt W. Zeller, Direktor des Keltenmuseum Hallein und Leiter des Österreichischen Forschungszentrums Dürrnberg tat sich als Ausgräber dutzender eisenzeitlicher Gräber hervor - hier bei der Freilegung des Grabes 145 im Jahre 1978 (Foto: Keltenmuseum Hallein/ÖFD).

ÖFD, das in den kommenden Jahren zahlreiche Not- und Forschungsgrabungen durchführte, zeugen der Gipfel der Grabungstätigkeit in den 1980er-Jahren und das konstant hohe Grabungsfortkommen bis in das neue Jahrtausend (Abb. 13). Im urgeschichtlichen Bestand bedeuteten auch nach dem Abschluss der Trassengrabungen der Jahre 1979- 
1982 die spektakulären Entdeckungen organischer Relikte in der latènezeitlichen „Gewerbesiedlung“ im Ramsautal, die erstmals 1982 durch Zeller gemacht wurden, einen maßgeblichen Objekt- und Erkenntniszuwachs. ${ }^{56}$ Neben den Grabungsarbeiten auf dem Dürrnberg wurde 2005 die Renovierung der Halleiner Stadtpfarrkirche archäologisch begleitet. ${ }^{57}$ Eine bereits seit 1981 existierende Stelle eines archäologischen Restaurators am Keltenmuseum Hallein, die von 1985 bis 2007 in das ÖFD übertragen wurde, half, den Fundmengen auch in konservatorischer Hinsicht Herr zu werden. Eine archäologische Zeichnerin am ÖFD trieb zwischen 1985 und 2004 die grafische Dokumentation der Objektbestände voran. Ab 1997 machte die nun auf zehntausende Objekte angewachsene archäologische Sammlung die Einrichtung einer weiteren, zunächst aus Fördermitteln, später durch eine Festanstellung bei der Stadtgemeinde Hallein finanzierte Restauratorenstelle am Keltenmuseum notwendig.

Mit Zeller übernahm 1989 erstmals ein akademisch ausgebildeter Fachwissenschaftler die Direktion des $\mathrm{Mu}$ seums, was sich auch in der zukünftigen Gestaltung und inhaltlichen Konzeption niederschlug. Durch die leitende Personalunion von Keltenmuseum und ÖFD war über Jahre eine Kompetenz wissenschaftlicher und musealer Arbeit gewährleistet, die Hallein und den Dürrnberg zu einem der Drehpunkte eisenzeitarchäologischer Forschung im europäischen Raum machte. Der Fokus der Dauerausstellung nahm als Alleinstellungsmerkmal die Prähistorie des keltischen Salzzentrums auf, dessen mittelalterlichneuzeitlicher Stadt- und Salinengeschichte dennoch eine weite Darstellungsfläche gelassen wurde (Abb. 14). Dieser Strategie war auch die Neukonzeption des Keltenmuseums verpflichtet, die zunächst 1994 durch die Anbindung des südlich an das Pfleggebäude anschließenden Wasnerhauses mittels eines modernen Stiegenhauses möglich wurde. In dem neu erschlossenen Gebäudetrakt konnten nun, insbesondere nach der grundlegenden Sanierung 2001/2002 Räumlichkeiten für Verwaltung, Forschungs- und Restaurierungsaufgaben sowie Depot- und Archivräume geschaffen werden. Sie dienen zudem der Aufstellung der umfangreichen Fachbibliothek, die mit einem Schwerpunkt auf metallzeitlicher Archäologie alle gängigen wissenschaftlichen Zeitschriften, Reihen und Monografien bereithält.

Die Konzentration von Logistik und Administration im Wasnerhaus bot der musealen Präsentation im Pflegtrakt mehr Raum und Möglichkeiten. 2004 wurde die dortige Situation den modernen Bedürfnissen eines

56 Zeller 1984. - Zeller 1988.

57 Zeller, Moser 2006.

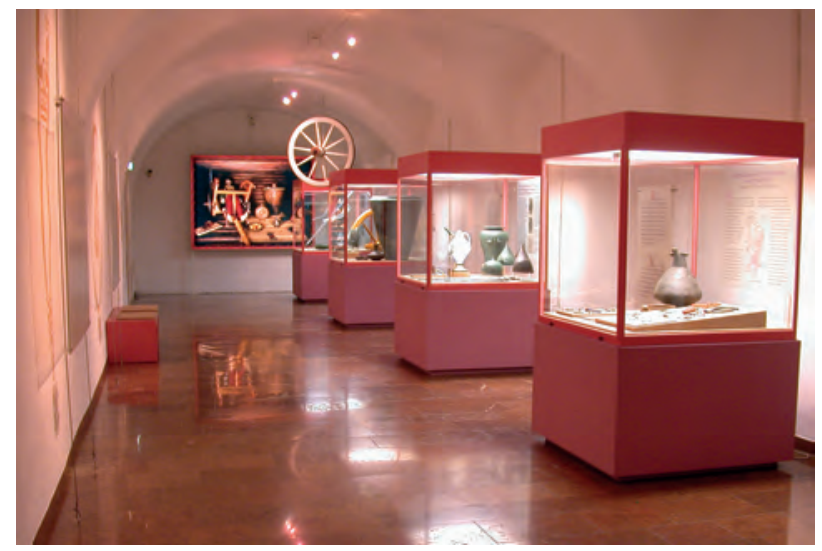

Abb. 14. Im Pflegtrakt der 1990er-Jahre nahm die eisenzeitliche Gräberlandschaft des Dürrnbergs breiten Raum ein (Foto: Keltenmuseum Hallein).

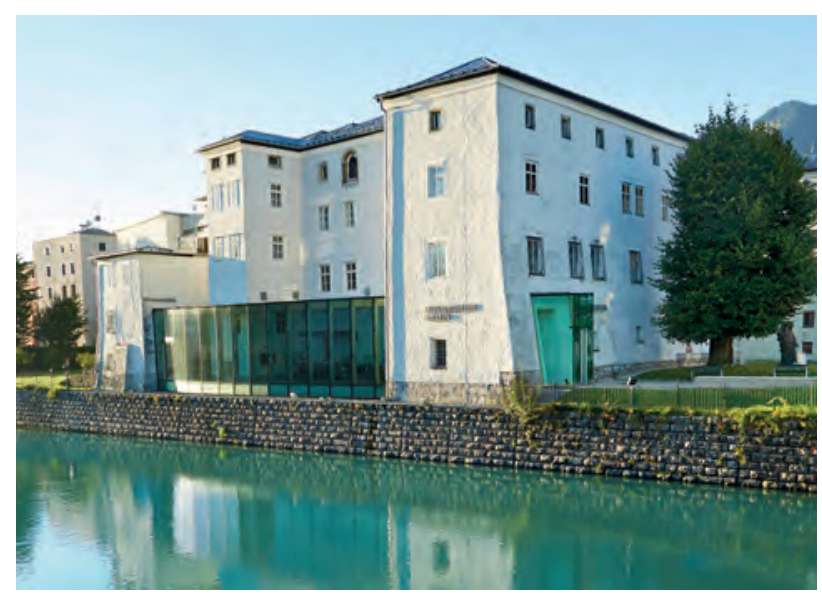

Abb. 15. Durch die bauliche Kombination des Pflegtraktes mit dem im Süden anschließenden „Wasnerhaus“ und die gläserne Optik des Eingangsbereiches erhielt das Keltenmuseum Hallein 2004 sein charakteristisches modernes Aussehen (Keltenmuseum Hallein, Foto: A. Hechenberger).

Museumsbetriebes durch eine umfassende bauliche und gestalterische Neukonzeption und einen neu gestalteten Kassen- und Eingangsbereich angepasst (Abb. 15). Die Präsentation der Stadt- und Salinengeschichte in Mittelalter und Neuzeit im 2. OG gruppierte sich um die mit einzigartigen Szenen der Bergarbeit des 18. Jhs. geschmückten „Fürstenzimmer" (Abb. 16)..$^{58}$ Besonders nach der Einstellung des produktiven Salzabbaus im Jahre 1989 trug das Keltenmuseum Hallein in verantwortungsbewusster Weise zur Sicherung des materiellen und kulturellen Erbes des nunmehr

58 ZELLER 1994 


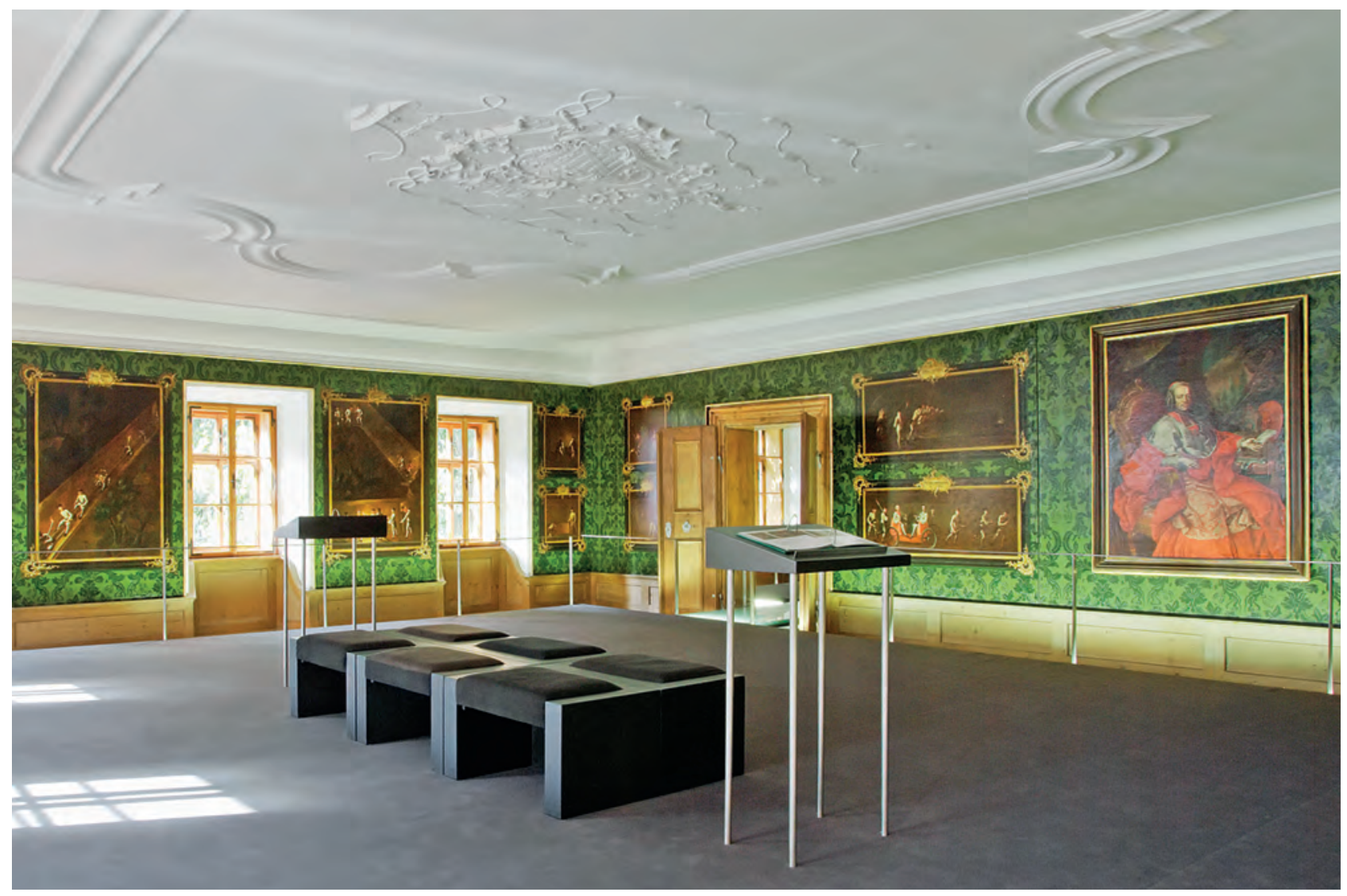

Abb. 16. In den „Fürstenzimmern“ im Obergeschoß des Pfleggebäudes stellen einzigartige Malereien den Solebergbau und das Halleiner Salzwesen des 18. Jhs. dar (Keltenmuseum Hallein, Foto: A. Hechenberger).

„historischen“ Halleiner Bergwesens bei. Dieses Engagement gipfelte zunächst in der Landesausstellung „Salz“ des Jahres 1994, die das Keltenmuseum auch räumlich mit dem Sudhaus und den Salzproduktionsstätten auf der benachbarten Pernerinsel durch eine spektakuläre Wege- und Stegführung über die Salzach hinweg verband..$^{59}$

Nach der Neugestaltung des Jahres 2004 bot das Keltenmuseum neben der eisenzeitlichen Archäologie des Dürrnbergs im 1. OG (Abb. 17) im Erdgeschoß Raum für die „Geschichte der Kelten“, Sonderausstellungen sowie Vorträge und Veranstaltungen. Die sehr detaillierte und umfassende Schausammlung brachte dem Keltenmuseum 2005 den Österreichischen Museumspreis ein. Die Begeisterung für die keltische Archäologie schlug sich 2006 in der Gründung des Museumsvereins „Celtic Heritage - Freunde des Keltenmuseum Hallein" nieder, der sich durch ideelle und finanzielle Unterstützung sowie ein abwechslungsreiches Angebot an Fachvorträgen oder Exkursionen für die Vermittlung und

59 Dopsch, Heuberger, Zeller 1994.

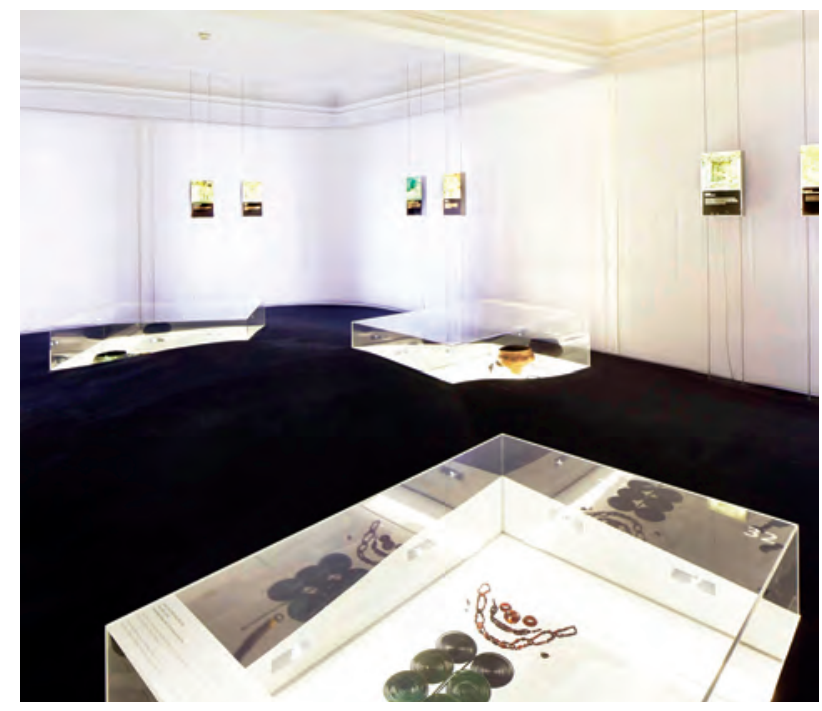

Abb. 17. Die Darstellung der Archäologie des Dürrnbergs im 2004 neu gestalteten Ausstellungsbereich folgt einem lichten Gestaltungskonzept (Keltenmuseum Hallein, Foto: A. Hechenberger). 
öffentliche Wahrnehmung des Museums und der Dürrnberger Kelten engagiert. ${ }^{60}$

Nicht zuletzt der wissenschaftlichen Expertise des ÖFD und seiner faktischen Nachfolgeorganisation, der „Dürrnbergforschung“, ist es zu verdanken, dass Hallein im Laufe der Jahre immer wieder Ort internationaler Archäologietagungen war. Ein ab 1976 jährlich stattfindendes „Keltensymposion des Lions-Clubs Hallein“, das mit Joachim Werner oder Georg Kossack renommierte Referenten vorweisen konnte, wurde anscheinend nach 1978 nicht fortgeführt. Die späteren Tagungen fanden in enger Kooperation mit anderen Museen, Forschungseinrichtungen oder Interessensverbänden, mitunter auch unter deren Ägide vor Ort statt. Sie setzten sich, dem programmatischen Namen des Museums gemäß, meist mit der eisenzeitlichen und keltischen Archäologie auseinander, thematisieren - und publizieren - aber stets auch allgemeine urgeschichtliche Forschungen. Das anlässlich des 50-Jahr-Jubiläums für das Jahr 2021 geplante Festkolloquium schließt den Kreis zur Eröffnung des Keltenmuseum Hallein „auf den Tag genau“, als zu deren Feier die Österreichische Arbeitsgemeinschaft für Ur- und Frühgeschichte in Hallein tagte:

15.-17. Oktober 1970 - 9. Jahrestagung der Österreichischen Arbeitsgemeinschaft für Ur- und Frühgeschichte „Hallstatt - Hallein. Probleme der älteren und jüngeren Eisenzeit“. - Kooperation: ÖAGU. ${ }^{61}$

26.-30. Oktober 1994 - Internationales ÖGUF-Symposium „Montanarchäologie. Salz - Kupfer - Gold“ - Kooperation: Salzburger Museum Carolino Augusteum.

7.-11. Oktober 1998 - Internationales Kolloquium „Dürrnberg und Manching - Wirtschaftsarchäologie im ostkeltischen Raum“ der Römisch-Germanischen Kommission Frankfurt/M. und des Vorgeschichtlichen Seminars der Philipps-Universität Marburg. ${ }^{62}$

7.-9. November 2011 - 4. Linzer Gespräche zur interpretativen Eisenzeitarchäologie „Die erfundenen Kelten - Mythologie eines Begriffes und seine Verwendung in Archäologie, Tourismus und Esoterik“ - Kooperation: Oberösterreichische Landesmuseen, University of Bangor (GB). ${ }^{63}$

12.-14. November 2015 - Internationale Tagung der AG Eisenzeit im West- und Süddeutschen Verband für Altertumskunde „Übergangswelten - Todesriten. Neue Forschungen zur Bestattungskultur der mitteleuropäischen

60 MÜнцвӧск 2015, 123-124.

61 Kaus 1971, 61-63, anschließend Resümees der Vorträge dieser Tagung: 64-114.

62 Dobiat, Sievers, Stöllner 2002.

63 Leskovar, Karl, Moser 2012.
Eisenzeit“. Kooperation: Salzburg Museum, Salzwelten Hallein. ${ }^{64}$

27.-30. Oktober 2016 - Internationales ÖGUF-Symposium „Mobilität und Kulturraum“ - Kooperation: Gesellschaft für Archäologie in Bayern e.V., Bayerisches Landesamt für Denkmalpflege, Bundesdenkmalamt, Institut für Urgeschichte und Historische Archäologie der Universität Wien.

9.-10. Mai 2019 - 23. Österreichische Tagung der Restauratorinnen und Restauratoren für archäologische Bodenfunde - Kooperation: Salzburg Museum. ${ }^{65}$

15.-17. Oktober 2021 - Festkolloquium „Die Kelten - Ursprung. Geschichte. Mythos“ - Kooperation: Archäologische Staatssammlung München, Bayerisches Landesamt für Denkmalpflege, Salzburg Museum.

Eine inhaltliche Differenzierung zog die Gründung des „Stille Nacht Museum Hallein“ nach sich, das sich seit 1993 im Wohnhaus des Komponisten und Organisten FranzXaver Gruber (1787-1863) befindet und als Teil des Keltenmuseums mitverwaltet und saisonal betrieben wurde. ${ }^{66}$ Es wurde im Vorfeld der Feierlichkeiten zum 200-jährigen Jubiläum des weltbekannten Weihnachtsliedes als Teil des Ensembles um den Kirchplatz zusammen mit dem „StilleNacht-Archiv“, der größten Text- und Dokumentsammlung zum Wirken Grubers, renoviert, neu gestaltet und 2018 wiedereröffnet. Das Stille-Nacht-Museum erhielt hierfür 2019 den „Anerkennungspreis zum Salzburger Museumsschlüssel“ der Salzburger Landesregierung.

\section{Museumspartner: Keltenmuseum Hallein und Salzburg Museum}

Ob der Präsenz und Allgegenwart Zellers im Museumsund Kulturleben der Stadt Hallein stellte sein überraschender Tod im Jahr 2009 einen tiefen und schmerzlichen Einschnitt in der Museums- und Forschungsarbeit dar. ${ }^{67}$ Die Kontinuität war zunächst durch die Übernahme der Leitung von Museum und ÖFD durch den Archäologen Stefan Moser gewährleistet, der sich seit einigen Jahren bei Feldforschungen auf dem Dürrnberg und der Entwicklung von Ausstellungen engagiert hatte. 2012 übernahm Florian K. Knopp die Leitung des Museums, die er bis dato innehat. Um die professionelle Strategie in den Bereichen Museumskonzeption und Administration sowie die öffentlichkeitswirksame Aufwertung und Vermarktung des Museums zu intensivieren, wurde im selben Jahr eine

\footnotetext{
64 WendLing et al. 2018.

65 Wendling 2019a.

66 Holzner, Knopp, Tober 2018.

67 Moosleitner 2009.
} 


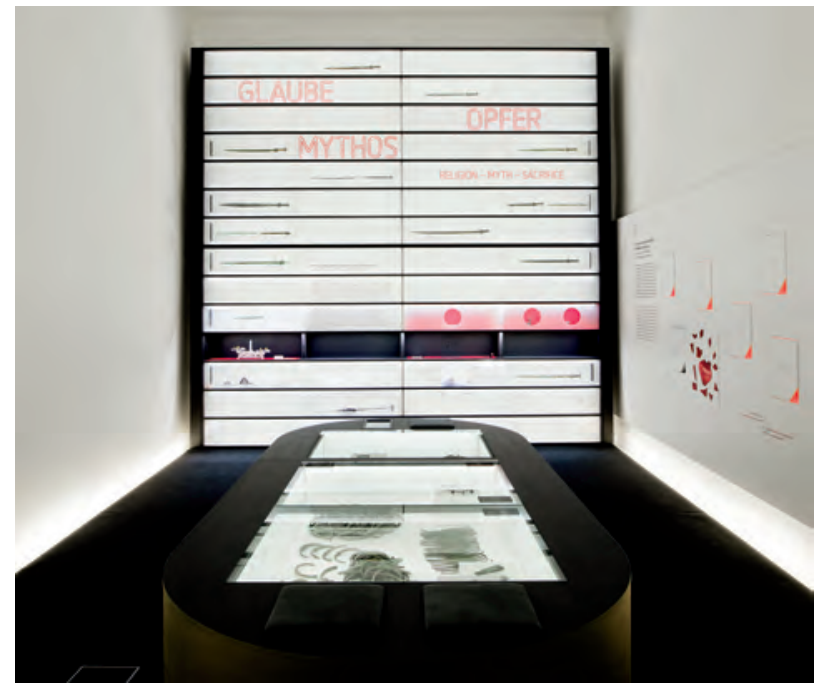

Abb. 18. Die aus der Kooperation zwischen Keltenmuseum Hallein und Salzburg Museum erwachsene Ausstellung „Zeitsprünge | Ursprünge - Reise in die Urgeschichte Salzburgs" verleiht dem Museum seit 2014 den Status eines Salzburger Zentralmuseums für Urgeschichte (Keltenmuseum Hallein, Foto: tolerian_archipicture).

Betriebsführungsvereinbarung mit der „Salzburg Museum $\mathrm{GmbH}^{\prime \prime}$ geschlossen. Die schon seit der Neuaufstellung des Museumsstatutes im Jahr 2000 etablierte engere Zusammenarbeit von Land Salzburg und Stadt Hallein, die sich nicht zuletzt in einem paritätisch besetzten Kuratorium niederschlägt, wird seither noch prägnanter umgesetzt und im konkreten Miteinander gelebt. ${ }^{68}$ Als plakatives Symbol dieser Kooperation gilt die weltberühmte Dürrnberger Schnabelkanne, die als Sammlungsobjekt des Fachbereiches Archäologie des Salzburg Museum seit April 2012 in der Dauerausstellung des Keltenmuseum Hallein präsentiert wird. Initialmoment der Zusammenarbeit bildete das kooperative Ausstellungsprogramm „Archäologie?! / Wirklich wichtig", das sich 2013 an den beiden Standorten in Hallein und Salzburg unterschiedlichen Facetten der vergangenen und modernen Altertumskunde sowie der Antikenrezeption widmete. ${ }^{69}$ Im Anschluss gelang es, den Museumsstandort Hallein durch die Einrichtung einer Dauerausstellung zu stärken, die die Präsentation des Keltenmuseums inhaltlich und zeitlich ergänzt und sich vornehmlich aus den archäologischen Sammlungsbeständen des Salzburg Museum speist. Die von kulturpolitischer und fachlicher Seite verfolgte Strategie, das Keltenmuseum als zentrales Archäologiemuseum für Urgeschichte des Landes Salzburg zu etablieren, ist hierdurch von nachhaltigem

68 Мӥнцвӧск 2015, 122-123.

69 HOChleitner 2013.

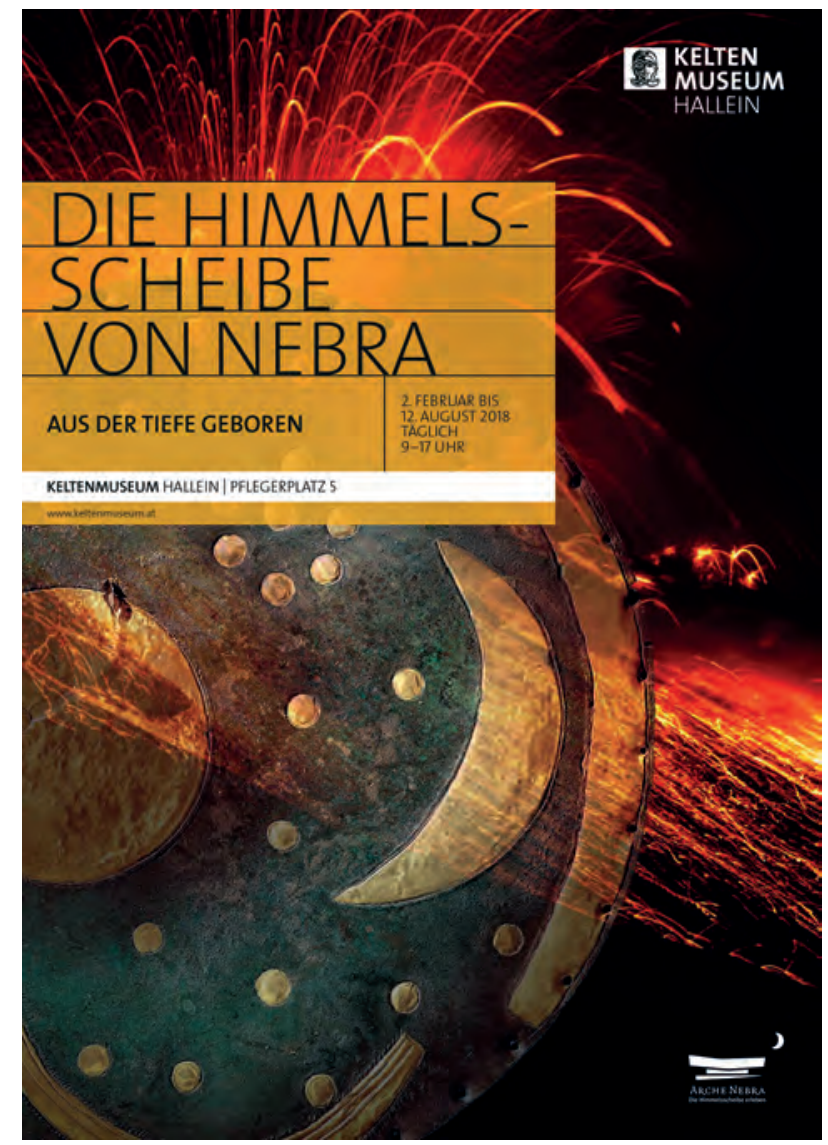

Abb. 19. Die 2017/18 in Kooperation mit dem Ausstellungszentrum „Arche Nebra“ (Sachsen-Anhalt, D) konzipierte Ausstellung „Die Himmelsscheibe von Nebra - Aus der Tiefe geboren“ war eine der erfolgreichsten Halleiner Sonderausstellungen (Grafik: Salzburg Museum/Keltenmuseum Hallein).

Erfolg gekrönt. Die Ausstellung „Zeitsprünge | Ursprünge - Reise in die Urgeschichte Salzburgs" setzt gestalterische Maßstäbe und bietet einen Überblick über 10.000 Jahre menschlicher Besiedlung in der Alpenregion um Salzach und Saalach (Abb. 18). ${ }^{70}$ Ein Handbuch gleichen Titels zur Salzburger Urgeschichte vertieft die museale Thematik und offenbart vielfältige Details zu archäologischen Befunden und Phänomenen. ${ }^{71}$

Seit jenen ersten Initiativen unter der Ägide des Salzburg Museum zeigt das Keltenmuseum jährlich ein bis zwei Sonderausstellungen, die neben dem Schwerpunktthema Archäologie auch stadtgeschichtlichen Themen nachgehen. So wurde etwa im Rahmen der Landesausstellung zur 200-jährigen Zugehörigkeit Salzburgs zu Österreich die Schau „SalzHochBurg Hallein - Ein Rohstoff, der

70 Wendiang 2015b.

71 WendLing 2018b. 

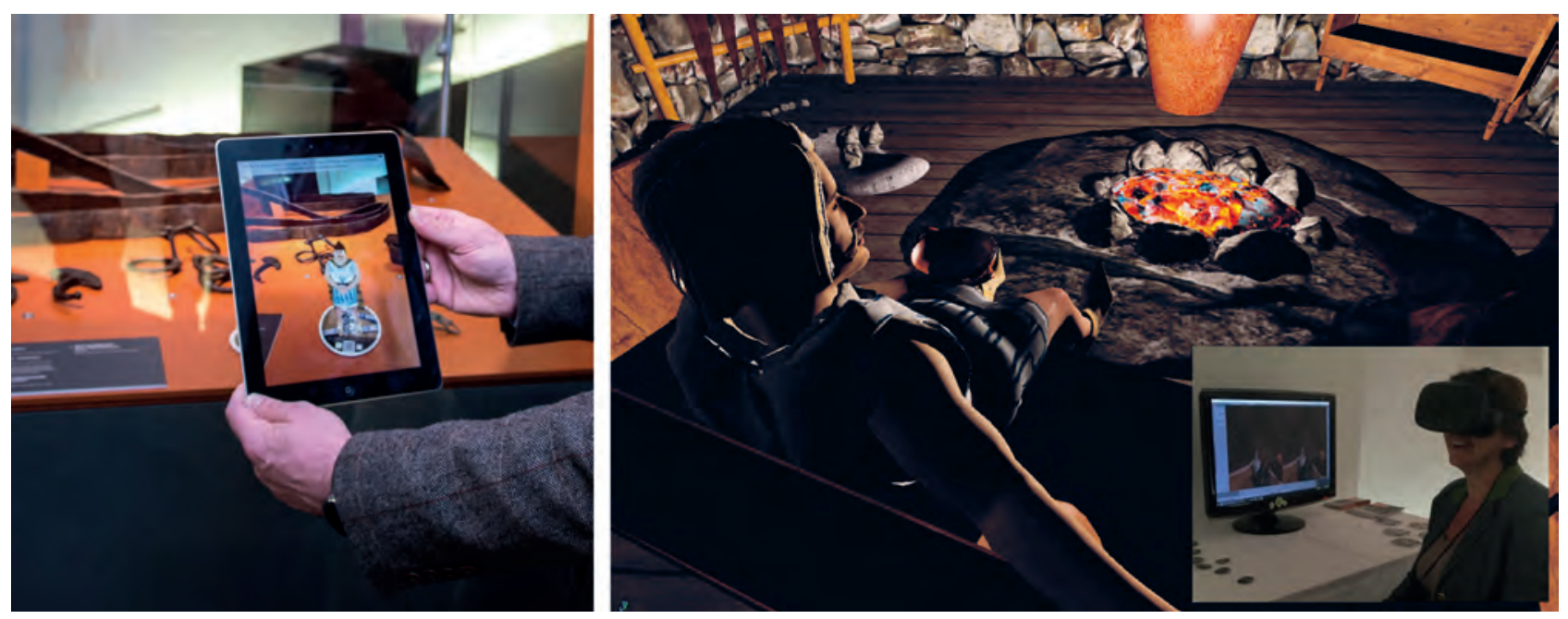

Abb. 20. Angmented reality und virtual reality-3D-Animationen bereichern die Möglichkeiten der modernen Kulturvermittlung (Keltenmuseum Hallein, Fotos: H. Wendling).

Land und Menschen prägte" gezeigt. ${ }^{72}$ Als Publikumsmagnet entpuppte sich jedoch die in Kooperation mit dem Ausstellungszentrum „Arche Nebra“ (Sachsen-Anhalt, D) konzipierte Ausstellung „Die Himmelsscheibe von Nebra - Aus der Tiefe geboren“, die das spektakuläre astronomische Instrument und Kultobjekt in den Mittelpunkt stellte (Abb. 19). ${ }^{73}$ Die Herkunft des für die Scheibe verwendeten Kupfers aus den Lagerstätten des Mitterberger Gebiets bei Bischofshofen ${ }^{74}$ zeigt den engen Bezug vermeintlich weit entfernter Regionen zur Salzburger Landesgeschichte und erlaubte so, die urgeschichtlichen Wirtschafts- und Sozialkontakte in ein modernes Ausstellungsprojekt zu projizieren.

Die wissenschaftliche Ausstellungskuratierung geht stets mit innovativen Vermittlungskonzepten und besonders auf Kinder zugeschnittenen museumspädagogischen Inhalten einher. Hervorzuheben sind hierbei zum Teil in weltweitem Ersteinsatz angewandte digitale Vermittlungsmedien. Der inner- und außerhalb des Museums anzutreffende virtuelle „sprechende Kelte“, der mit Smartphone und Spezial-App „aktiviert“ werden kann, integriert die Technologien der augmented reality in den analogen Museumsraum. Dieser wird zudem durch den Einsatz virtueller 3D-Technologien um eine parallele Realität bereichert, die das Erlebnis einer Zeitreise suggeriert (Abb. 20). Unterstützend kommen hierbei neueste digitale

72 Baumgartner et al. 2017.

73 WendLing 2018c. - WendLing 2018d.

74 Diverse Beiträge in Stöllner, Oeggl 2015. - Pernicka, Lutz, STÖLLNER 2016.
Dokumentationsmethoden zum Tragen, die die kostbaren Exponate materialschonend und en détail interaktiv erfahrund analysierbar machen. ${ }^{75}$

In jüngerer Zeit intensiviert das Keltenmuseum Hallein die kooperativen Beziehungen $\mathrm{zu}$ anderen Museen und Interessensverbänden. 2018 wurde es Mitglied des Vereins „KeltenWelten e.V.“, eines Zusammenschlusses von bedeutenden Stätten mit keltischer Geschichte in Deutschland und dem benachbarten Ausland - tatsächlich ist Hallein erster Partner in dieser „,internationalen Dimension“. Um den neu gewonnenen Kontakt zu Kommunen, auf deren Gebiet Fundorte der keltischen Epoche liegen, zu Tourismusverbänden, Museen, Forschungseinrichtungen und Landesämtern für Denkmalpflege unmittelbar zu festigen, konzipierte das Keltenmuseum auf Basis einer Wanderausstellung des Vereins eine umfassende Schau „KeltenWelten - Archäologie einer geheimnisvollen Kultur“ mit Leihgaben vom Keltenmuseum Hochdorf, dem Landesdenkmalamt Baden-Württemberg und der HessenArchäologie. ${ }^{76}$ Als herausragende Kombination an $\mathrm{Ob}$ jekten zog die Besucherinnen und Besucher vor allem die Gegenüberstellung der originalen Dürrnberger Bronzeschnabelkanne und ihres Pendants aus dem „Fürstengrab“ vom Glauberg in Kopie in ihren Bann. Darüber hinaus wird zukünftig auch die Zusammenarbeit im Netzwerk „Iron Age Europe“ vorangetrieben, zu dem etwa das kelten römer museum Manching, das schweizerische Laténium, das französische Centre archéologique Européen Bibracte oder

75 WendLING, Rabsilber 2017.

76 WeNDLING 2019b. - WeNDLING 2019c. 
die Archäologische Staatssammlung München gehören. Die hier entwickelte Sonderausstellung „Bilderwelt der Kelten“ wird 2021 eröffnet werden.

\section{Archäologie am Museum - die Dürrnbergforschung}

Die äußeren und finanziellen Zwänge, die 2012 zur Auflösung des ÖFD führten, ließen zunächst Befürchtungen aufkommen, den archäologischen Forschungen auf dem Dürrnberg könnte ein allzu abruptes Ende beschieden sein. Die Verantwortlichen der Gemeinde Hallein mit ihrer in besonderer Weise für das Keltenmuseum und den Dürrnberg engagierten Kulturstadträtin und Kuratoriumsvorsitzenden Eveline Sampl-Schiestl sowie die Stellen des Landes Salzburg begegneten der Gefahr in Zeiten allfälliger Kürzungen im Kulturbereich indes mit überlegtem und verantwortungsvollem Handeln. Grundlegendes zur Kontinuität trug in dieser problematischen Übergangsphase der wissenschaftliche Beirat des Keltenmuseum Hallein bei, der unter dem Vorsitz Friedrich Steinkellners (Wissenschaftsförderung Land Salzburg) und Walter Irlingers (Bayerisches Landesamt für Denkmalpflege München) die wissenschaftliche Vakanz überbrückte. Er verhalf dem Keltenmuseum Hallein und der assoziierten, neu etablierten „Dürrnbergforschung“ weiterhin zu dem ihnen gebührenden Status in der Geschichte des Landes und in der internationalen Wissenschaftslandschaft. Die seit 2012 bestehende Betriebsführungsvereinbarung zwischen Keltenmuseum Hallein und der Salzburg Museum GmbH schuf hierfür auch in personeller Hinsicht Synergien, durch die vor allem die wissenschaftliche Expertise im Keltenmuseum weiterhin gewährleistet wird. Das Engagement der Dürrnbergforschung schlägt sich insbesondere in der für lange Zeit virulenten, nun umso resoluter forcierten Aufarbeitung der Dürrnberger Grabfunde nieder, die in einer international beachteten Publikationstätigkeit gipfeln. In der Abteilung „Gräberarchäologie“ der Monografienreihe „Dürrnberg-Forschungen“ konnten seit 2012 neun, teilweise doppelbändige Abhandlungen zu einzelnen Dürrnberger Gräberfeldern und anderen Fundkomplexen vorgelegt werden. ${ }^{77}$ Mehrere Aufarbeitungsprojekte zu weiteren Gräberarealen, etwa zwei Analysen der sogenannten „Nordgruppe“ befinden sich in unterschiedlichen Stadien der Bearbeitung. ${ }^{78}$ Mit der Veröffentlichung der mit 110 Grablegen umfangreichsten Nekropole im „Eislfeld“ ist man der abschließenden, in enger interdisziplinärer Verbindung zur Anthropologie und Archäozoologie am Naturhistorischen

77 Zuletzt Lavelle, Stöllner 2019. - Boenke 2020. - Franke, WiLTSCHKE-SCHROTTA 2021.

78 Zeller 2003b.
Museum Wien konzipierten Gesamtdarstellung der Dürrnberger Bestattungskultur einen entscheidenden Schritt näher gekommen. ${ }^{79}$ Parallel hierzu werden siedlungsarchäologische Grabungen durchgeführt, die in Verknüpfung mit den seit 1999 durch das Deutsche Bergbaumuseum und die Ruhr-Universität Bochum stattfindenden montanarchäologischen Untersuchungen ein umfassendes Bild der Dürrnberger Salzmetropole zeichnen sollen. ${ }^{80}$ So gelang es, nach der grundlegenden Arbeit von Ernst Pucher und mehreren Folgeanalysen neuerlich einen diachronen Bestand an Tierknochenresten zu analysieren, der das Konsum- und Wirtschaftsgebaren der eisenzeitlichen Bevölkerung offenlegte. ${ }^{81}$ Die Vorlage aller keltischen und römischen Münzfunde vom Dürnberg und seiner Umgebung sowie die archäometrische Analyse mehrerer Goldbarrengussformen bieten numismatische und wirtschaftshistorische Erkenntnisse. ${ }^{82}$ Modernste naturwissenschaftliche Analysen eisenzeitlicher Gold- und Korallenobjekte, die in Kooperation mit dem Competence Center Archaeometry Baden-Württemberg in Tübingen, dem Curt-Engelhorn-Zentrum Archäometrie in Mannheim und der Archäologischen Staatssammlung München durchgeführt werden, geben Einblick in eisenzeitliche Ressourcennutzung und Sozialkontakte über große Distanzen. ${ }^{83}$ Archäologische Ausgrabungen und geophysikalische Prospektionen, die meist im Zuge von Bauprojekten durchgeführt werden, bereichern das Bild der eisenzeitlichen Siedlungs- und Sepulkralkultur. ${ }^{84} \mathrm{Da}$ die aktuellen Ergebnisse stets auch in die museale Projektplanung und Kuratierung einfließen, hat sich die Verzahnung von Museum und Wissenschaft neuerlich konsolidiert. Überraschungsfunde, wie der fast 40 Jahre unentdeckt im Depot schlummernde Henkel einer griechischen Glanztonschale, die um 450 v. Chr. ihren Weg an den Fuß des Dürrnberg fand, illustrieren das nachhaltige wissenschaftliche Potential der Sammlung (Abb. 21). ${ }^{85}$ Sie wird auch in Zukunft die Position des Keltenmuseum Hallein als zentraler Ausstellungs- und Forschungsschwerpunkt zur eisenzeitlichen Archäologie Europas sichern.

Vor diesem musealen und wissenschaftlichen Aktivitätsspektrum hat sich die Hoffnung, der die Halleiner

79 Rabsilber, Wendling, Wiltschke-Schrotta 2017.

80 Zur Montanarchäologie zusammenfassend jüngst mehrere Beiträge in STÖLLNER, OEGGL 2015. - Zur Dürrnbergforschung zuletzt WENDLING, IRLINGER 2020.

81 Horvath, Wendling 2020. - Grundlegend Pucher 1999.

82 SChachinger, WENDling 2019.

83 Bente et al. in Vorb.

84 Preinfalk, Preinfalk, Wendling 2017. - Kirchmayr, WendLING im Druck.

85 WeNDLING in Vorb. 


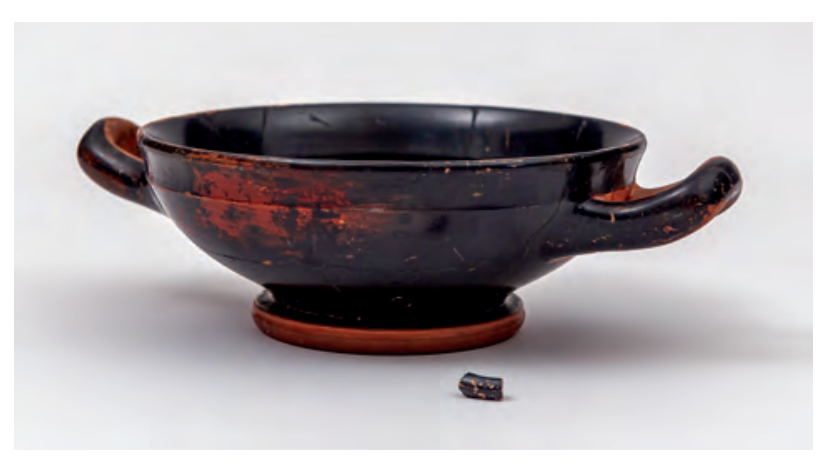

Abb. 21. Der Fund eines Jahrzehnte unentdeckt im Depot des Keltenmuseum Hallein schlummernden Henkelbruchstücks griechischer Importkeramik fügt sich der vollständigen Kylix aus Grab 44\#2 an (Keltenmuseum Hallein, Foto: C. Kossmann).

Zeitung zur Eröffnung 1970 Ausdruck gab, das Museum möge „von Jahrzehnt zu Jahrzehnt an Umfang und Bedeutung gewinnen und $\mathrm{zu}$ einem wertvollen Hort und Studienplatz für europäische Kunst- und Kulturgeschichte werden “ ${ }^{86}$ uneingeschränkt erfüllt. Heute präsentiert sich das Haus als Salzburger Zentralmuseum für Urgeschichte und kooperiert mit den renommiertesten Fundort- und Archäologiemuseen sowie Forschungsinstitutionen Europas. Im Verbund mit der wissenschaftlichen Erschließung der Dürrnberger Salzmetropole wird dem überregionalen $\mathrm{Pu}$ blikum die Faszination eines Zentrums der keltischen Welt und die sinnstiftende Rolle der Archäologie vermittelt.

Mit dieser Perspektive ist gleichfalls ein Auftrag für kommende Dekaden verbunden, dem sich das Keltenmuseum Hallein durch die fruchtbare Zusammenarbeit von Stadtgemeinde Hallein und Land Salzburg selbstbewusst stellen kann. In musealer Hinsicht tragen hierzu eine Ausweitung der Depotflächen und der bereits initiierte Ausbau des Dachgeschoßes des Pfleggebäudes zum „KurtZeller-Forum“ bei, das für zukünftige großformatige Ausstellungsprojekte unabdingbaren Raum bieten wird. Hierdurch wird sich das Museum noch prägnanter als einer der maßgeblichen Archäologiestandorte und Fundortmuseen Österreichs in Szene setzen und der Präsentation der keltischen Kultur am Dürrnberg und über die Region hinaus eine Plattform bieten. Die Grundlage hierfür kann auch in Zukunft nur eine fundierte und intensive archäologische Forschung am Stand- und Fundort gewährleisten. Hiervon wird nach der Aufarbeitung und umfassenden Interpretation der eisenzeitlichen Bestattungskultur zunächst der seit 2004 existierende Dauerausstellungsbereich profitieren, der adaptiert und aktualisiert

86 Halleiner Zeitung 1970b. werden wird. Die Dürrnbergforschung wird hiernach indes nicht innehalten, sondern die antike Lebensrealität der Salzmetropole mit neuesten Methoden umso intensiver analysieren. Hierzu sind mit den archäometrischen Projekten, die über die Untersuchungen von Gold- und Korallenartefakten angestoßen wurden, ideale Grundlagen bereitet. Die interdisziplinären Forschungsprojekte werden ausgeweitet und nehmen zunächst die naturwissenschaftliche Analyse des Glasschmucks - neben Manching besitzt der Dürrnberg das größte Konvolut an jüngerlatènezeitlichen Glasarmringen Europas - in das Blickfeld. ${ }^{87}$ Isotopen- und Spurenelementmessungen versprechen nicht nur hierbei wichtige Erkenntnisse zur Wirtschaftsarchäologie und zum urgeschichtlichen Technologietransfer. Die hiermit aufs engste verknüpfte Kommunikation der Menschen kann mittlerweile durch modernste Verfahren, etwa Strontiumisotopie oder die Möglichkeiten der aDNA-Analyse individualisiert und konkretisiert werden. Auch hierzu sind erste kooperative Projekte mit Forschungsinstitutionen aus ganz Europa angelaufen. Für archäometrische Forschungen zu Nahrungsrückständen in eisenzeitlichen Grabbeigabengefäßen und den damit verbundenen Fragen zum urgeschichtlichen Konsum- und Kultverhalten bietet der Dürrnberg mit tausenden Gefäßen in fast 400 Gräbern der Phasen Ha D1/2 bis Lt C eine unvergleichliche Datenbasis. Sie zu nutzen stellt gleichermaßen Herausforderung und einmalige Chance einer modernen interdisziplinären Archäologie dar, der sich die Dürrnbergforschung auch in Zukunft verpflichtet fühlt.

\section{Danksagung}

Ich danke Dr. Walter Irlinger (München) und Margarethe Kirchmayr MA (Hallein) für die kritische Durchsicht des Manuskripts und wertvolle Hinweise. Dr. Anna Holzner (Hallein) und Benjamin Huber MA (Hallein) recherchierten freundlicherweise Nachrichten- und Bildmaterial im Stadtarchiv Hallein.

\section{Literatur}

BARTH 1996

F. E. BARTh (Hrsg.), Der Spurensucher: Zum 200. Geburtstag von Johann Georg Ramsauer. Katalog des Oberösterreichischen Landesmuseums N. F. 93, 2. Auflage, Linz 1996.

BAUMGARTNER et al. 2017

J. Baumgartner, J. F. Schatteiner, A. Strobl, W. WinterstelLER, SalzHochBurg Hallein: Ein Rohstoff, der Land und Menschen prägte. Schriftenreihe des Stadtarchivs Hallein 3, Salzburg 2017.

Bente et al. in Vorb.

K. Bente, C. Berthold, H. Wendling, R. Wirth, M. Ende, A. Gerdes, R. P. Selvam, Coral and Glass from Iron Age Dürrnberg: Archaeological and Scientific Approaches to Material, Colour, and Meaning, in Vorbereitung.

87 BRAND 2002. 


\section{BoENKe 2020}

N. Boenke, Die Analyse botanischer Großreste aus dem Salzbergbau am Dürrnberg bei Hallein: Eine Untersuchung zu Ressourcen, Umwelt und Ernährung. Dürrnberg-Forschungen 12, Abteilung Naturwissenschaften, Rahden/Westf. 2020.

BRAND 2002

C. Brand, Graphitton und Glas: Studien zur keltischen Keramikund Armringproduktion vor dem Hintergrund Dürnberger Siedlungsfunde. In: C. Dobiat, S. Sievers, T. Stöllner (Hrsg.), Dürnberg und Manching: Wirtschaftsarchäologie im ostkeltischen Raum. Akten des Internationalen Kolloquiums in Hallein/ Bad Dürrnberg vom 7. bis 11. Oktober 1998. Kolloquien zur Vor- und Frühgeschichte 7, Bonn 2002, 107-116.

Dobiat, Sievers, StÖLlner 2002

C. Dobiat, S. Sievers, T. Stöllner (Hrsg.), Dürrnberg und Manching: Wirtschaftsarchäologie im ostkeltischen Raum. Akten des Internationalen Kolloquiums in Hallein/Bad Dürrnberg vom 7. bis 11. Oktober 1998. Kolloquien zur Vor- und Frühgeschichte 7, Bonn 2002.

Dopsch, Heuberger, Zeller 1994

H. Dopsch, B. Heuberger, K. Zeller (Red.), Salz (Ausstellungskatalog, 7. Salzburger Landesausstellung, Pernerinsel und Keltenmuseum Hallein, 30. April bis 30. Oktober 1994). Salzburg 1994.

Dückher von Haslau 1666

F. Dückher von Haslau, Saltzburgische Chronica: Das ist, Beschreibung deß Lands/Stifftung und denckwürdiger Geschichten / deß Hoch-löbl. Ertz-Stiffts Saltzburg, Mit schönen Kupfferstücken deß Lands / dessen fürnehmsten Städt und Oerter / auch aller Bischöff und Ertz-Bischoffen-Wappen. Salzburg 1666.

EGgERT 2012

M. K. H. Eggert, Prähistorische Archäologie: Konzepte und Methoden, 4. Auflage. Tübingen - Basel 2012.

Franke, WiltschKe-SChrotTA 2021

R. Franke, K. Wiltschke-Schrotta, Der Dürnberg bei Hallein: Die Gräbergruppe am Steigerhaushügel. Dürrnberg-Forschungen 13, Abteilung Gräberkunde, Rahden/Westf. 2021.

Halleiner Zeitung 1970a

Unterrichtsminister Dr. Gratz eröffnet Keltenmuseum, Halleiner Zeitung, 19. Jg., Nr. 41, 15.10.1970, 1.

Halleiner Zeitung 1970b

Das Keltenmuseum - ein Halleiner Schatzhaus, Halleiner Zeitung, 19. Jg., Nr. 42, 22.10.1970, 1.

HANSEN 2001

S. Hansen, Von den Anfängen der prähistorischen Archäologie: Christian Jürgensen Thomsen und das Dreiperiodensystem, Prähistorische Zeitschrift 76/1, 2001, 10-23.

HeLl 1914

M. HeLl, Neue Funde am Dürrnberg bei Hallein, Mitteilungen der k. k. Zentral-Kommission für Denkmalpflege 13/3. Folge, 1914, 220-222.

HeLl 1916

M. HeLL, Vorgeschichtliche Funde vom Dürnnberg bei Hallein, Wiener Prähistorische Zeitschrift 3, 1916, 57-70.

HeLl 1926

M. Held, Neue Beiträge zur Vor- und Frühgeschichte des Dürnberges bei Hallein, Mitteilungen der Anthropologischen Gesellschaft in Wien 56, 1926, 320-345.

HeLl 1927a

M. HeLl, Neue Gräber vom Dürrnberg bei Hallein, Mitteilungen der Anthropologischen Gesellschaft in Wien 57, 1927, [113]-[114].

\section{HeLl 1927b}

M. Hell, Exkursion am 13. September 1926 auf den Dürrnberg bei Hallein, Mitteilungen der Anthropologischen Gesellschaft in Wien 57, 1927, [176].

HeLl 1929

M. HeLl, Neue Grabfunde der Hallstatt- und Latènezeit vom Dürrnberg bei Hallein, Mitteilungen der Anthropologischen Gesellschaft in Wien 59, 1929, 155-180.

HeLl 1931

M. HeLl, Eine metallzeitliche Hirschhornaxt aus Salzburg, Wiener Prähistorische Zeitschrift 18, 1931, 152-154.

HeLl 1934

M. HeLl, Keltische Kopfapplike vom Dürrnberg bei Hallein in Salzburg, Germania 18, 1934, 189-193.

HeLl 1970

M. Hell, Archäologisches Tagebuch, Heft 199, Eintrag vom 17.10.1970. Salzburg Museum, FB Archäologie, Archiv.

HOCHLEITNER 2013

M. Hochleitner (Hrsg.), Archäologie in Salzburg. Archäologie in Salzburg 7, Salzburg 2013.

Holzner, Knopp, Tober 2018

A. Holzner, F. Knopp, B. Tober, Stille Nacht Museum Hallein: Dem Komponisten begegnen: Franz Xaver Gruber. Schriftenreihe des Stadtarchivs Hallein 4, Salzburg - Hallein 2018.

Horvath, WendLing 2020

J. Horvath, H. Wendling, Tierhaltung und Fleischkonsum auf dem eisenzeitlichen Dürrnberg: Die Faunenreste der Grabung „Hochbichl/Emco 2015“, Annalen des Naturhistorischen Museums in Wien $122 \mathrm{~A}, 2020,5-25$.

JACOBSTHAL 1935

P. Jaсовsтнац, Keltenkunst, Atlantis: Länder, Völker, Reisen 7/3, 1935, 186-191.

JACOBSTHAL 1944

P. Jacobsthal, Early Celtic Art. Oxford 1944.

KANZLER 1912

G. J. KanZler, Die Stadt Hallein und ihre Umgebung: Ein Wegweiser für Einheimische und Fremde. Hallein 1912.

KaUs 1971

K. Kaus, Bericht über die 9. Jahrestagung der Österreichischen Arbeitsgemeinschaft für Ur- und Frühgeschichte in Hallein in der Zeit vom 15. bis 17. Oktober 1970, Mitteilungen der Österreichischen Arbeitsgemeinschaft für Ur- und Frühgeschichte 22/2, 1971, 61-63.

KirchmaYr, Wending im Druck

M. Kirchmayr, H. Wending, KG Dürnberg, SG Hallein: Grabungsbericht, Fundberichte aus Österreich 58/2019, im Druck.

\section{KLEIN 1961}

H. Klein, Der Fundort des „Mannes im Salz“, Mitteilungen der Gesellschaft für Salzburger Landeskunde 101, 1961, 139-141.

KLoSE 1934

O. KLOSE, Die Schnabelkanne vom Dürrnberg bei Hallein, Salzburg, Wiener Prähistorische Zeitschrift 21, 1934, 83-107.

KOLLER 2016

F. KOLLer, Der Bestand der Originalurkunden im Stadtarchiv/Keltenmuseum Hallein, Salzburg-Archiv 36, 2016, 33-43.

KosSACK 1995

G. Kossack, Nachruf: Ludwig Pauli 18.3.1944-12.7.1994, Bayerische Vorgeschichtsblätter 60, 1995, 1-14. 
KRENN 1961

E. KRENN, Bericht über die Jahrestagung 1960 der Österr. Arbeitsgemeinschaft für Ur- und Frühgeschichte in Hallein u. SalzburgStadt in der Zeit vom 26. Mai bis 29. Mai 1960, Mitteilungen der Österreichischen Arbeitsgemeinschaft für Ur- und Frühgeschichte 12/1, 1961, 32-33.

LAVELle, Stöllner 2019

R. Lavelle, T. Stöllner, Der Dürrnberg bei Hallein: Die Gräbergruppe und die Siedlungs- und Ritualbefunde am Simonbauernfeld. Dürrnberg-Forschungen 11, Abteilung Gräberkunde, Rahden/Westf. 2019.

LesKovar, KARL, MOSER 2012

J. Leskovar, R. Karl, S. Moser (Hrsg.), Interpretierte Eisenzeiten: Die erfundenen Kelten: Mythologie eines Begriffes und seine Verwendung in Archäologie, Tourismus und Esoterik. Tagungsbeiträge der 4. Linzer Gespräche zur interpretativen Eisenzeitarchäologie. Studien zur Kulturgeschichte Oberösterreichs 31, Linz 2012.

MAIER 1974

F. MAIER, Gedanken zur Entstehung der industriellen Großsiedlung der Hallstatt- und Latènezeit auf dem Dürrnberg bei Hallein, Germania 52, 1974, 326-374.

MOOSLEITNER 1977

F. Moosleitner, Die Erforschung des Dürrnberges: Ein Überblick über die seit mehr als 150 Jahren andauernden Grabungen im Bereich der prähistorischen Salzmetropole, Salzburger Museumsblätter 38, 1977, 26-31.

MoOsleitner 1985

F. Moosleitner, Die Schnabelkanne vom Dürrnberg: Ein Meisterwerk keltischer Handwerkskunst. Salzburg 1985.

Moosleitner 2009

F. Moosleitner, In memoriam Mag. Kurt Wolfgang Zeller, Direktor des Keltenmuseums Hallein und Leiter des Österreichischen Forschungszentrums Dürrnberg, Archäologie Österreichs 20/1, 2009, 64-65.

Moosleitner, Pauli, Penninger 1974

F. Moosleitner, L. Pauli, E. Penninger, Der Dürrnberg bei Hallein II: Katalog der Grabfunde aus der Hallstatt- und Latènezeit. Münchner Beiträge zur Vor- und Frühgeschichte 17, München 1974

Much 1882

M. Much, Bericht über die II. Versammlung österreichischer Anthropologen und Urgeschichtsforscher am 12., 13. und 14. August 1881 zu Salzburg, Mittheilungen der Anthropologischen Gesellschaft in Wien 12, 1882,1-76.

Much, PetTer, Bouchal 1906

M. Much, A. Petter, L. Bouchal, II. Äusserer Verlauf der IV. gemeinsamen Versammlung der Deutschen und Wiener anthropologischen Gesellschaft, zugleich XXXVI. allgem. Versammlung der Deutschen anthropologischen Gesellschaft in Salzburg, Mitteilungen der Anthropologischen Gesellschaft in Wien 36, 1906, [72]-[83].

MüнLвÖск 2015

A. Müнцвӧск, Hallein: Stadt im Wandel der Zeit. Hallein 2015.

PAUli 1978

L. PAuli, Der Dürrnberg bei Hallein III, 1/2: Auswertung der Grabfunde. Münchner Beiträge zur Vor- und Frühgeschichte 18, München 1978 .
Penninger 1960a

E. Penninger, Ein Fürstengrab der Latènezeit vom Dürrnberg bei Hallein, Mitteilungen der Gesellschaft für Salzburger Landeskunde 100, 1960, 1-14.

PenNinger $1960 \mathrm{~b}$

E. Penninger, Vorläufiger Bericht über die neugefundenen latènezeitlichen Gräber vom Dürrnberg bei Hallein, Germania 38, 1960, 353-363.

Penninger 1963

E. Penninger, Das Stadtmuseum Hallein, Salzburger Museumsblätter 26/1-2, N. F. 4, 1963, 4-6.

Penninger 1972

E. Penninger, Der Dürrnberg bei Hallein I: Katalog der Grabfunde aus der Hallstatt- und Latènezeit: Erster Teil. Münchner Beiträge zur Vor- und Frühgeschichte 16, München 1972.

PenNinger 1976

E. Penninger, Das Keltenmuseum Hallein, Österreichs Museen stellen sich vor 7, 1976, 41-47.

Penninger 1980

E. Penninger, Geschichte der archäologischen Forschung auf dem Dürrnberg. In: L. PAuli (Red.), Die Kelten in Mitteleuropa: Kultur, Kunst, Wirtschaft (Ausstellungskatalog, Salzburger Landesausstellung im Keltenmuseum Hallein, 1. Mai - 30. Sept. 1980). Salzburg 1980, 150-158.

Pernicka, Lutz, Stöllner 2016

E. Pernicka, J. Lutz, T. Stöllner, Bronze Age copper produced at Mitterberg, Austria, and its distribution, Archaeologia Austriaca 100, 2016, 19-55.

Preinfalk, Preinfalk, Wendling 2017

A. Preinfalk, F. Preinfalk, H. Wendling, KG Dürnberg, SG Hallein: Grabungsbericht, Fundberichte aus Österreich 54/2015, 2017, 328-331.

Pucher 1999

E. Pucher, Archäozoologische Untersuchungen am Tierknochenmaterial der keltischen Gewerbesiedlung im Ramsautal auf dem Dürrnberg (Salzburg). Dürrnberg-Forschungen 2, Abteilung Naturwissenschaft, Rahden/Westf. 1999.

Rabsilber, Wendling, Wiltschke-Schrotta 2017

T. Rabsilber, H. Wendling, K. Wiltschke-Schrotta, Der Dürrnberg bei Hallein: Die Gräbergruppe im Eislfeld. Dürrnberg-Forschungen 10, Abteilung Gräberkunde, Rahden/Westf. 2017.

RICHTER 1879

E. Richter, Die Funde auf dem Dürenberg bei Hallein, Mitteilungen der Gesellschaft für Salzburger Landeskunde 19, 1879, 184-191.

RiCHTER 1880

E. Richter, Die Funde auf dem Dürenberg bei Hallein II: Nachträge, Mitteilungen der Gesellschaft für Salzburger Landeskunde 20, 1880, 91-96.

RICHTER 1881

E. RichteR, Verzeichnis der Fundstellen vorhistorischer und römischer Gegenstände im Herzogthume Salzburg, Mitteilungen der Gesellschaft für Salzburger Landeskunde 21, 1881, 90-97.

vON SACKEN 1868

E. von SACKen, Das Grabfeld von Hallstatt in Oberösterreich und dessen Alterthümer. Wien 1868.

SALzburger NACHRICHTEN 1970

Keltenmuseum schon vor der Eröffnung zu klein, Salzburger Nachrichten, 26. Jg., Nr. 237, 14.10.1970, 5. 
Schachinger, Wendling 2019

U. Schachinger, H. WendLing, Numismatik einer Salzmetropole: Fundmünzen und Edelmetallguss der Latène- und Römerzeit auf dem Dürrnberg und in Hallein, Bayerische Vorgeschichtsblätter 84, 2019, 171-210.

SChatTeiner, StÖLLner 2001

J. F. Schatteiner, T. Stöllner, Männer im Salz - Verunglückte Knappen: Grubenunglücke und Arbeitsunfälle im Dürrnberger Salzbergbau, Der Anschnitt 53/2-3, 2001, 71-79.

SEETHALER 1831

J. A. Seethaler, Die allerersten Celtischen und Römischen Alterthümer am Dürrnberg und zu Hallein an der Salza in Verbindung mit ihren Salinen. Manuskript o. O. 1831.

SMCA 1898

Jahresbericht des städtischen Museum Carolino-Augusteum zu Salzburg. Salzburg 1898.

SONNTAg 2000

F. Sonntag, Johann Andreas Seethaler - ein Heimatforscher um 1800, Oberösterreichische Heimatblätter 54, 2000, 83-90.

Stöllner, Oeggl 2015

T. Stöllner, K. OegGl (Hrsg.), Bergauf Bergab: 10.000 Jahre Bergbau in den Ostalpen (Ausstellungskatalog, Deutsches BergbauMuseum Bochum). Veröffentlichungen aus dem Deutschen Bergbau-Museum Bochum 207, Bochum 2015.

Tennengauer Zeitung 1951

Verein „Heimatmuseum Hallein“ gegründet, Tennengauer Zeitung, 1. Jg., Nr. 24, 24.8.1951, 2.

Tiefengraber, WiltschKe-SChrotTA 2014

G. Tiefengraber, K. Wiltschke-Schrotta, Der Dürrnberg bei Hallein: Die Gräbergruppe Hexenwandfeld. Dürrnberg-Forschungen 7, Abteilung Gräberkunde, Rahden/Westf. 2014.

VOLKSFREUND 1930

Das Halleiner Heimatmuseum: Zu seiner Eröffnung am 24. August 1930, Volksfreund, 40. Jg., Nr. 33, 16.8.1930, 3-4.

WAGNER 1906a

H. F. Wagner, Aus Halleins Vergangenheit: III. Zur Chronik der Stadt, Volksfreund, 6. Jg., Nr. 9, 3.3.1906, 5.

WAGNER 1906b

H. F. WAgner, Aus Halleins Vergangenheit: IV. Zur Chronik des Dürrnberges (nebst Nachträgen über Hallein): Der Dürrenberg bei Hallein nächst Salzburg, Volksfreund, 16. Jg., Nr. 36, 7.9.1906, 6.

WAGNER 1913

H. F. WAGNER, Sebastian Wimmer: Ein Erinnerungsblatt, Volksfreund, 24. Jg., Nr. 7, 15.2.1913, 2-4.

WENDLING 2013

H. Wendling, 200 Jahre Archäologie am Dürnberg: Ein Fundort zwei Länder - drei Sammlungen. In: M. Hochleitner (Hrsg.), Archäologie in Salzburg. Archäologie in Salzburg 7, Salzburg 2013, 49-63.

WENDLING 2015a

H. Wendling, Natur und Kultur über und unter Tage, Archäologie in Deutschland 4/2015, 2015, 70-71.

WeNDLING 2015b

H. WendLing, Zeitsprünge zu den Ursprüngen: Die Salzburger Urgeschichte im Keltenmuseum Hallein, Archäologie Österreichs 26/2, 2015, 52-55.
WeNDLING 2016

H. Wendling, Wechselnde Identitäten: Bergbau und Archäologie im Salzburger Geschichtsbewusstsein. In: Bischof. Kaiser. Jedermann: 200 Jahre Salzburg bei Österreich, 2: Erzähl mir Salzburg! (Begleitband, Salzburger Landesausstellung, 2016), Jahresschrift des Salzburg Museum 58/2, 2016, 33-46.

WENDLING 2018a

H. Wendling, Frühkeltische Kunst: Vergangene Bildwelten Vergessene Mythen. In: R. Kastler, F. Lang, H. Wendling (Hrsg.), Faber Salisburgi. Festschrift für Wilfried K. Kovacsovics zum 65. Geburtstag. ArchaeoPlus 10, Salzburg Studien 18, Salzburg 2018, 359-379.

WENDLING 2018b

H. Wendling, Zeitsprünge | Ursprünge: Reise in die Urgeschichte Salzburgs: Ein Handbuch der urgeschichtlichen Archäologie einer Alpenregion. Salzburg 2018.

WENDLING 2018c

H. Wending, Himmelsscheibe aus Salzburger Kupfer, Bayerische Archäologie 2/2018, 2018, 52-53.

WENDLING 2018d

H. Wendling, Der Mitterberg - Wiege der Europäischen Metallurgie, Salzburger Museumsblätter 79/6, 2018, 5.

WeNDLING 2019a

H. Wending, Restaurierung international im Keltenmuseum Hallein, Journal - Magazin des Österreichischen Restauratorenverbandes 12, 2019, 61-63.

WeNDLING 2019b

H. Wendling, Geheimnis Kelten, Bayerische Archäologie 2/2019, 2019, 54-55.

WENDLING 2019c

H. Wenduing, Blattkrone \& Mistelkult: Religion, Symbole und Herrschaft der frühen Kelten, Salzburg Museum - Das Kunstwerk des Monats 32, 2019, B1. 374.

WENDLING in Vorb.

H. Wendling, Greek and Italic imports at Early Iron Age Dürrnberg: a study in transalpine communication, Germania, in Vorbereitung.

WENDLING, IRLINGER 2020

H. Wendling, W. Irlinger, Die Dürrnbergforschung: Archäologie einer eisenzeitlichen Salzmetropole, Archäologie Österreichs 28/1-2/2017, 2020, 2-16.

WeNDLING, RABSILBER 2017

H. Wending, T. Rabsilber, Schnabelkanne 3D - Digitale Dokumentation, Analyse, Archivierung und Präsentation keltischer Kunst. In: R. KARL, J. LESKOvAR (Hrsg.), Interpretierte Eisenzeiten: Fallstudien, Methoden, Theorie. Tagungsbeiträge der 7. Linzer Gespräche zur interpretativen Eisenzeitarchäologie. Studien zur Kulturgeschichte Oberösterreichs 47, Linz 2017, 255-268.

WENDLING et al. 2018

H. Wendling, M. Augstein, J. Fries-Knoblach, K. Ludwig, R. Schumann, C. Tappert, P. Trebsche, J. Wiethold (Hrsg.), Übergangswelten - Todesriten: Forschungen zur Bestattungskultur der europäischen Eisenzeit. Beiträge zur internationalen Tagung der AG Eisenzeit in Hallein 2015 und zur Sitzung der AG Eisenzeit während des 8. Deutschen Archäologiekongresses in Berlin 2014. Beiträge zur Ur- und Frühgeschichte Mitteleuropas 86, Langenweißbach 2018. 
WiLlvonSEDER 1931

K. Willvonseder, Neues vom „Mann im Salz“, Wiener Prähistorische Zeitschrift 18, 1931, 154-156.

WiLLVONSEDER 1960a

K. Willvonseder, Keltische Kunst in Salzburg (Ausstellungskatalog, 30. Sonderausstellung des Salzburger Museums Carolino Augusteum, Salzburg, Museumspavillon im Mirabellgarten, Juni-September 1960, Hallein, Oktober 1960). Schriftenreihe des Salzburger Museums Carolino Augusteum 2, Salzburg 1960.

WiLLVONSEDER $1960 \mathrm{~b}$

K. W. Willvonseder, Ausstellungen, Jahresschrift Salzburger Museum Carolino Augusteum 6, 1960, 167-180.

ZELLER 1980

K. W. Zeller, Die neuen Grabungen auf dem Dürrnberg - Techniken und Ergebnisse. In: L. PAuli (Red.), Die Kelten in Mitteleuropa: Kultur, Kunst, Wirtschaft (Ausstellungskatalog, Salzburger Landesausstellung im Keltenmuseum Hallein, 1. Mai - 30. Sept. 1980). Salzburg 1980, 159-181.

ZELLER 1984

K. W. Zeller, Latènezeitliche Gewerbebetriebe auf dem Dürrnberg bei Hallein. In: O.-H. FREY, H. RoTH (Hrsg.), Studien zu Siedlungsfragen der Latènezeit. Veröffentlichungen des Vorgeschichtlichen Seminars Marburg, Sonderband 3, Marburg 1984, 199-214.

ZELLER 1988

K. W. Zeller, Neue keltische Gewerbebauten auf dem Dürrnberg bei Hallein, Salzburg-Archiv 6, 1988, 5-22.

ZELLER 1990

K. W. Zeller, Fünf Jahre Forschungszentrum Dürrnberg, SalzburgArchiv 10, 1990, 17-24.

ZELLER 1994

K. W. Zeller, Die Fürstenzimmer. In: H. Dopsch, B. HeuberGER, K. Zeller (Red.), Salz (Ausstellungskatalog, 7. Salzburger Landesausstellung, Pernerinsel und Keltenmuseum Hallein, 30. April bis 30. Oktober 1994). Salzburg 1994, 175-238.

ZELLER 1997

K. W. ZelleR, Das österreichische Forschungszentrum Dürrnberg, Archäologie Österreichs 8/2, 1997, 4-20.

ZELLER 2001

K. W. Zeller, Der Dürrnberg bei Hallein: Ein Zentrum keltischer Kultur am Nordrand der Alpen. Hallein 2001.

ZELLER 2002

K. W.Zeller, Dürrnberger Gräber als Spiegel der Fernbeziehungen. In: G. Schnekenburger (Hrsg.), Über die Alpen: Menschen Wege - Waren. ALManach 7/8, Stuttgart 2002, 191-203.

ZELLER 2003a

K. W. Zeller, Luxus und Fernhandelsgüter für den keltischen Adel auf dem Dürrnberg bei Hallein. In: C. SchwanZar, G. WinkLER, J. Leskovar (Hrsg.), Worauf wir stehen: Archäologie in Oberösterreich. Bibliothek der Provinz 1, Weitra 2003, 275-282.

ZELLER 2003b

K. W. Zeller, Die „Nordgruppe“ - ein latènezeitliches Gräberfeld am Fuße des Putzenkopfes auf dem Dürrnberg bei Hallein, Fundberichte aus Österreich 42, 2003, 525-558.

ZELler, Moser 2006

K. W.Zeller, S. Moser, KG Hallein, SG Hallein, VB Hallein, Fundberichte aus Österreich 45, 2006, 744-748.
Holger Wendling

Salzburg Museum Alpenstraße 75 5020 Salzburg

Österreich

$\varepsilon$

Keltenmuseum Hallein

Pflegerplatz 5

5400 Hallein

Österreich

bolger.wendling@salzburgmuseum.at

(D) orcid.org/0000-0003-0295-0248 
